\title{
Resistance training with different repetition duration to failure: Effect on hypertrophy, strength and muscle activation
}

\author{
Lucas Túlio Lacerda ${ }^{1,2,3,4}$, Rodrigo Otávio Marra-Lopes ${ }^{1}$, Marcel Bahia Lanza ${ }^{1,5}$, Rodrigo César Ribeiro Diniz ${ }^{1}$, \\ Fernando Vitor Lima ${ }^{1}$, Hugo Cesar Martins-Costa ${ }^{1,4}$, Gustavo Ferreira Pedrosa ${ }^{1,6}$, André Gustavo Pereira Andrade ${ }^{1}$ \\ , Armin Kibele ${ }^{7}$, Mauro Heleno Chagas ${ }^{\text {Corresp. } 1}$ \\ ${ }^{1}$ Weight Training Laboratory, School of Physical Education, Physiotherapy and Occupational Therapy, Universidade Federal de Minas Gerais, Belo \\ Horizonte, Minas Gerais, Brazil \\ 2 Department of Physical Education and Sports, Centro Federal de Educação Tecnológica, Belo Horizonte, Minas Gerais, Brazil \\ 3 Universidade do Estado de Minas Gerais, Divinópolis, Minas Gerais, Brazil \\ 4 Department of Physical Education, Pontifícia Universidade Católica de Minas Gerais, Belo Horizonte, Minas Gerais, Brazil \\ 5 Department of Physical Therapy and Rehabilitation, School of Medicine, University of Maryland, Maryland, Baltimore, United States \\ 6 Aeronautical Instruction and Adaptation Centre, Brazilian Air Force, Lagoa Santa, Minas Gerais, Brazil \\ 7 Institute for Sports and Sport Science, University of Kassel, Mönchebergstraße, Kassel, Germany \\ Corresponding Author: Mauro Heleno Chagas \\ Email address: mauroufmg@hotmail.com
}

Background. This study investigated the effects of two 14-week resistance training protocols with different repetition duration (RD) performed to muscle failure (MF) on gains in strength and muscle hypertrophy as well as on normalized electromyographic (EMG) amplitude and force-angle relationships.

Methods. The left and right legs of ten untrained males were assigned to either one of the two protocols (2-s or 6-s RD) incorporating unilateral knee extension exercise. Both protocols were performed with 3-4 sets, $50-60 \%$ of the one-repetition maximum (1RM), and 3 min rest. Rectus femoris and vastus lateralis muscles cross-sectional areas (CSA), maximal voluntary isometric contraction (MVIC) at $30^{\circ}$ and $90^{\circ}$ of knee flexion and 1RM performance were assessed before and after the training period. In addition, normalized EMG amplitude-angle and force-angle relationships were assessed in the $6^{\text {th }}$ and $39^{\text {th }}$ experimental sessions.

Results. The 6-s RD protocol induced larger gains in MVIC in the $30^{\circ}$ of knee angle measurement than the 2-s RD protocol. Increases in MVIC in the $90^{\circ}$ of knee angle and 1RM were indifferent between the 2-s and 6-s RD protocols. For the rectus femoris muscle growth, inconclusive changes were found across the ten subjects. In contrast, the 2-s RD protocol may have resulted in superior vastus lateralis muscle hypertrophy. Moreover, different normalized EMG amplitude-angle and force-angle values were detected between protocols over most of the angles analyzed.

Conclusion. Performing longer RD could be a more appropriate strategy to provide greater gains in isometric maximal muscle strength at shortened knee positions. However, similar maximum dynamic strength and muscle hypertrophy gains would be provided by protocols with different RD. 
1 Resistance training with different repetition duration to

2 failure: Effect on hypertrophy, strength and muscle

3 activation

Lucas Túlio de Lacerda ${ }^{1,2,3,4}$, Rodrigo Otávio Marra-Lopes ${ }^{1}$, Marcel Bahia Lanza ${ }^{1,5}$, Rodrigo César Ribeiro Diniz ${ }^{1}$, Fernando Vitor Lima ${ }^{1}$, Hugo Cesar Martins-Costa ${ }^{1,4}$, Gustavo Ferreira Pedrosa $^{1,6}$, André Gustavo Pereira Andrade ${ }^{1}$, Armin Kibele ${ }^{7}$, Mauro Heleno Chagas ${ }^{1}$

${ }^{1}$ Weight Training Laboratory, School of Physical Education, Physiotherapy and Occupational Therapy, Universidade Federal de Minas Gerais, Belo Horizonte, Minas Gerais, Brazil

${ }^{2}$ Department of Physical Education and Sports, Centro Federal de Educação Tecnológica, Belo Horizonte, Minas Gerais, Brazil

${ }^{3}$ Universidade do Estado de Minas Gerais, Divinópolis, Minas Gerais, Brazil

${ }^{4}$ Department of Physical Education. Pontifícia Universidade Católica de Minas Gerais, Belo Horizonte, Minas Gerais, Brazil

${ }^{5}$ Department of Physical Therapy and Rehabilitation, School of Medicine, University of Maryland, Maryland, Baltimore, United States

${ }^{6}$ Aeronautical Instruction and Adaptation Centre, Brazilian Air Force, Lagoa Santa, Minas Gerais, Brazil

${ }^{7}$ Institute for Sports and Sport Science, University of Kassel, Mönchebergstraße, Kassel, Germany

Corresponding Author:

Mauro Chagas

Av. Antônio Carlos, 6627, Belo Horizonte, Minas Gerais, 31270-901, Brazil

Email address: mauroufmg@hotmail.com

Abstract

Background. This study investigated the effects of two 14-week resistance training protocols with different repetition duration (RD) performed to muscle failure (MF) on gains in strength and muscle hypertrophy as well as on normalized electromyographic (EMG) amplitude and forceangle relationships.

Methods. The left and right legs of ten untrained males were assigned to either one of the two protocols (2-s or 6-s RD) incorporating unilateral knee extension exercise. Both protocols were performed with 3-4 sets, $50-60 \%$ of the one-repetition maximum (1RM), and 3 min rest. Rectus femoris and vastus lateralis muscles cross-sectional areas (CSA), maximal voluntary isometric contraction (MVIC) at $30^{\circ}$ and $90^{\circ}$ of knee flexion and 1RM performance were assessed before 
and after the training period. In addition, normalized EMG amplitude-angle and force-angle relationships were assessed in the $6^{\text {th }}$ and $39^{\text {th }}$ experimental sessions.

Results. The 6-s RD protocol induced larger gains in MVIC in the $30^{\circ}$ of knee angle measurement than the 2-s RD protocol. Increases in MVIC in the $90^{\circ}$ of knee angle and 1RM were indifferent between the 2-s and 6-s RD protocols. For the rectus femoris muscle growth, inconclusive changes were found across the ten subjects. In contrast, the 2-s RD protocol may have resulted in superior vastus lateralis muscle hypertrophy. Moreover, different normalized EMG amplitude-angle and force-angle values were detected between protocols over most of the angles analyzed. Conclusion. Performing longer RD could be a more appropriate strategy to provide greater gains in isometric maximal muscle strength at shortened knee positions. However, similar maximum dynamic strength and muscle hypertrophy gains would be provided by protocols with different RD.

\section{Introduction}

Repetition duration (RD) is an important feature of a resistance training program (ACSM, 2009) influencing the strength gains and hypertrophy (i.e. quadriceps femoris muscles) (Chaves et al., 2020; Tanimoto \& Ishii, 2006). Nevertheless, the systematic effect of the RD on resistance training is not yet fully understood (Davies et al., 2017; González-Badillo et al., 2014). It has been reported that measurements on isokinetic devices showed poor training and sports specificity (e.g. reduced ecological validity) and the lack of equalization of resistance training protocols would be some of the limitations presented by studies that investigated the influence of RD (González-Badillo et al., 2014). Moreover, the absence of registration and/or poor control over the RD, especially during protocols to muscle failure (MF), may hamper its meaning for the effectiveness isoinertial exercises (González-Badillo et al., 2014). Hence, RD control and comparability between training protocols must be considered to be mandatory for a proper understanding of the $\mathrm{RD}$ effect in a resistance training program.

A meta-analysis on the RD effect on muscle hypertrophy (including only studies with protocols performed to MF) concluded that similar muscle hypertrophy responses may be observed when performing RD between 0.5-s and 8-s (Schoenfeld et al., 2015). This result suggests that a wide RD range may be employed in order to produce muscle hypertrophy. However, in addition to RD, the meta-analysis also included studies with variations in the load intensity (Schuenke et al., 2012), and studies with training protocols performed until MF (Tanimoto \& Ishii, 2006) or not. Consequently, the results of the meta-analysis cannot be attributed to the manipulation of RD only. Previous studies have suggested that muscle strength and hypertrophy are predominantly influenced by the load intensity [(e.g. percentage of one repetition maximum - \%1RM)] (Lasevicius et al., 2018) and by the RD (González-Badillo et al., 2014; Tanimoto \& Ishii, 2006). Therefore, given that different variables combined may simultaneously influence the chronic responses induced by strength training (ACSM, 2009), the effect of RD only within a resistance training to MF while controlled for the load intensity remains unknown. 
Another aspect to be considered in studies investigating the impact of RD on muscle hypertrophy relates to the use of different assessment instruments (e.g. biopsy, magnetic resonance imaging or ultrasound) and assessment locations on the muscle (e.g. $50 \%$ of the muscle femur length). The cross-sectional area (CSA) is a valid measure of muscle hypertrophy. However, single-point measurements somewhere along the muscle length may not adequately represent the entire muscle hypertrophic response (Noorkoiv et al., 2014). Thus, a CSA analysis including several assessment locations along the muscle length may possibly provide a more accurate depiction of the muscle hypertrophic response in comparison with a specific region along the muscle length (Noorkoiv et al., 2014) and, therefore, a more accurate analysis of hypertrophy gains after resistance training programs as well.

Protocols performed with different RDs enforce different mechanical responses, with higher force values for shorter RD (Sampson et al., 2014; Tanimoto \& Ishii, 2006). As a consequence, different gains in muscle hypertrophy may be induced through resistance training with different RDs (Gonzalez et al., 2016). However, Sampson and Groeller (2016) showed that a resistance training protocol performed with faster movements (shorter RD) produced similar muscle hypertrophy when compared to a protocol with slower movements (longer RD). Given that the faster movements were not executed with the maximum number of repetitions, the results by Sampson and Groeller (2016) remain inconclusive about the RD effect during resistance training to MF. In addition, the protocols with faster movements were performed with a time under tension (TUT, up to three times shorter than the protocol with slower movement) and also higher training volume. The similar muscle hypertrophy observed between protocols reinforce the argument about the impact of mechanical tension (force applied by external resistance to the musculature) to induce adaptations. In this sense, the higher training volume and TUT performed during the longer RD protocol was probably a balance factor in relation to the greater magnitude of mechanical tension observed during protocols with shorter RD (verified by higher peak force values) (Sampson et al., 2014), inducing to similar muscle hypertrophy.

In addition, it has been shown that protocols with shorter RD performed to MF presented higher degrees of normalized electromyographic (EMG) amplitude compared to protocols performed with longer RD (Sakamoto \& Sinclair, 2012). An increase in the EMG amplitude is associated either with a higher motor unit recruitment or an increase in the firing frequency of the motor units (Hunter et al., 2004). Both factors would contribute to chronic adaptations associated with resistance training (Schoenfeld et al., 2014). Therefore, considering that the magnitude of the mechanical tension and EMG amplitude would be determinant factors of neuromuscular adaptations (Gehlert et al., 2015), protocols with shorter RD performed to MF (consequently higher number of repetitions) should theoretically provide superior responses of muscle hypertrophy when compared to protocols performed with longer RD.

As above-mentioned, protocols incorporating different RDs and repetition numbers provide different mechanical (Sampson et al., 2014) and neurophysiological responses (Lacerda et al., 2016; Sampson et al., 2014). As reported for muscle hypertrophy, it has been presented that these factors can influence muscle strength throughout resistance training. In this sense, the 
119

120

121

122

123

124

125

126

127

128

129

130

131

132

133

134

135

136

137

138

139

140

141

142

143

144

145

146

147

148

149

150

151

152

153

154

155

156

157

review by Davies et al. (2017) verified only a trend for larger gains in muscular strength (measured by 1RM performance) for protocols with shorter RDs and moderate intensities (60$79 \%$ 1RM) compared to longer RDs. Unfortunately, protocols with exercises leading to MF were not considered in this review. Moreover, the 1RM test does not provide information on maximum force values in different joint angles. In particular, the 1RM test fails to provide information about maximal force values in specific sections of the ROM where a mechanical disadvantage may possibly occur to explain the different adaptations to RT (van den Tillaar et al., 2012). As a consequence, maximum voluntary isometric contractions (MVIC) should be analyzed across a range of different joint angles to properly understand the effects of different RDs (Alegre et al., 2014).

In the past, studies showed that different RDs evolved to different force-angle relationships across the ROM. This was particularly true for the beginning and the end of the muscle actions (Sampson et al., 2014; Tanimoto \& Ishii, 2006). Protocols with shorter RDs require faster movements. Therefore, they lead to larger peak forces at the beginning of the concentric action (e.g., lengthened position during knee extension) compared to protocols with longer RDs (Sampson et al., 2014). At the end of the ROM (e.g., shortened position during knee extension), a decrease in force is observed when faster movements are performed. In contrast, protocols with longer RDs come along with less variation in the force response throughout the ROM, while larger force values appear at the end of the concentric actions (Sampson et al., 2014; Tanimoto \& Ishii, 2006). All in all, varied strategies to apply force throughout the concentric action incorporating different RDs may promote different increases in maximal isometric strength at specific points across the ROM. As a consequence, it was the aim of this study to compare the effects of two protocols with different RDs performed to MF on measures of maximal strength (1RM and MVIC) and muscle hypertrophy (CSA). A secondary aim was to compare the effects of these RD strategies on features of the normalized EMG amplitude-angle and force-angle relationships during both protocols execution. Based on our previous arguments, we hypothesized that larger increases in the 1RM and the CSA would be induced by a protocol with shorter RDs. In addition, the MVIC gains were expected to be different in specific areas across the ROM. In particular, larger forces were expected for faster training protocol at $90^{\circ}$ of knee flexion (stretched position) and for slower protocol at $30^{\circ}$ of knee flexion (shortened position).

\section{Materials \& Methods}

Study design

In the present study, a repeated measures design was adopted. Given that the unilateral exercise model reduces inter-subject variability, it can serve to increase statistical power, as well as reduce the time and cost of a study (MacInnis et al., 2017). Volunteers performed two resistance training protocols with two RDs (2-s or 6-s RD protocol) for 14 weeks. The left and the right legs were randomly assigned and balanced for limb dominance to either one of the protocols. Pre and post-test measures included: CSA, MVIC and 1RM tests. To assess the lower limb 
158

159

160

161

162

163

164

165

166

167

168

169

170

171

172

173

174

175

176

177

178

179

180

181

182

183

184

185

186

187

188

189

190

191

192

193

194

195

196

dominance voluntaries were asked to answer the following question: "If you would shoot a ball on a target, which leg would you use to shoot the ball?"

In session 1, limb dominance was determined, volunteers were familiarized with all the procedures, and training protocols were assigned to each limb. In the next session, ultrasound images were recorded to determine rectus femoris and vastus lateralis muscles CSA. The strength tests (MVIC and 1RM) were conducted in sessions 3 and 4 separated by at least $48 \mathrm{~h}$. Next, subjects trained from sessions 5 to 39 for a total of 14 weeks and five training sessions per week. The training sessions were separated by at least $24 \mathrm{~h}$. For each week, subjects trained their left or right either on days 1, 3, and 5, or on days 2 and 4 in an alternating way. Through this training schedule, a minimum of $48 \mathrm{~h}$ inter session rest was provided for each leg. In sessions 6 and 39 (for each protocol), the rectus femoris and vastus lateralis EMG amplitude were assessed through surface EMG while participants performed their respective training protocols. In session 40 , separated between 72 and $120 \mathrm{~h}$ from the last training session, the post-test ultrasound measurements were conducted similar to session 2. Finally, in session 41, the MVIC and 1RM post-tests were executed for both lower limbs.

\section{Participants and Ethics}

The sample size calculation was performed by using the software G.Power for Windows version 3.1.9.7 (Düsseldorf, Germany) and by following the guidelines proposed by Beck (2013), with a priori statistical power $(1-\beta)=0.80$, effect size $(f)=0.57$ and $5 \%$ significance level. Number of groups $=2$ (protocols); number of measures $=2$ (pre-post); correlation between measures $=0.90$; sphericity $=1$. For the sample size calculation, we used the absolute AST values of the vastus lateralis muscle from a previous study from our own laboratory, which was carried out with an experimental design similar to the present study (Lacerda et al., 2019). Ten males aged between 18 and 30 years (mean $\pm \mathrm{SD}$ : age $=23.1 \pm 4.63$ years; body height $=1.72 \pm 0.07 \mathrm{~m}$; body mass $=$ $68.4 \pm 9.46 \mathrm{~kg}$; body fat percentage $=14.03 \pm 6.56 \%$ ) participated in this study.

The inclusion criteria for participation were: (1) no resistance training during the last six months; (2) no functional limitations that could influence the 1RM test or the training protocols; and (3) no use of pharmacological substances or ergogenics supplements, and no other modes of resistance exercise during the study period. Subjects were informed about the study aims, procedures, and risks prior to signing an informed consent form. The ethics committee of the Federal University of Minas Gerais approved this study (approval number: 79108117.5.0000.5149), which complied with the Declaration of Helsinki. Additionally, each subject was instructed not to engage in any physical activity immediately before the testing sessions and to maintain the same diet before each session.

Experimental Session 1(anthropometric measurements)

After receiving information about the goals and the purpose of the study and giving written consent, the volunteers answered the Physical Activity Readiness Questionnaire (PAR-Q). Next, they were submitted to an anamnesis examining possible limitations related to the study participation. In addition, body height, mass, and fat percentage (skinfold thickness) 
197

198

199

200

201

202

203

204

205

206

207

208

209

210

211

212

213

214

215

216

217

218

219

220

221

222

223

224

225

226

227

228

229

230

231

232

233

234

235

measurements were conducted. As a next step, volunteers were positioned on a seated knee extension machine (Master, Minas Gerais, Brazil) while maintaining a hip angle of $110^{\circ}$ (angle between the backrest and the equipment seat). For measurement purposes, the lateral epicondyle of the femur was aligned with the rotational axis of the device and the pad of the device placed approximately $3 \mathrm{~cm}$ above the medial malleolus. These positions were registered for future replication in the subsequent tests and training sessions. All test sessions were held at the same time of the day for each volunteer.

\section{Experimental Sessions 2 and 40 (CSA - ultrasound measurements)}

During these sessions, ultrasound images were recorded for the CSA analysis of the rectus femoris and vastus lateralis muscles. The acquisition procedure for the CSA images was conducted as described by Noorkoiv et al. (2010). Initially, volunteers remained in a dorsal decubitus position on a stretcher for 15 minutes. During this period, the anterior regions of both thighs were marked to identify the reference points for the ultrasound image acquisition. Next, the major trochanters and lateral epicondyles of the femurs were identified, and femur length was measured (Figure 1A). From the proximal end of thigh, 40, 50, 60, and 70\% of femur length were identified and marked on volunteer's skin by using a tape measure and a pachymeter positioned parallel to the intercondylar line. Then, a line with a microporous adhesive tape was attached $2 \mathrm{~cm}$ from each percentage point on the thigh (Figure 1B) to delimitate the probe guide area for the ultrasound image acquisition (Figure 1C). Finally, the distances between the intercondylar line and each percentage point on the thighs were recorded for post-test replication. The procedures used to acquire images in the pre-test were the same for the post-test session ( $40^{\text {th }}$ session). The latter was started no earlier than $72-120 \mathrm{~h}$ following the last training session.

\section{- PLEASE INSERT FIGURE 1 HERE -}

An ultrasound device (MindRay DC-7, Shenzhen, China) was used in an extended-fieldof-view mode with a $4 \mathrm{~cm}$ linear transducer. The equipment was configured with $10 \mathrm{MHz}$ frequency, an acquisition rate of 21 frames/s, a depth for the image capture ranging from 7.7 and $9.7 \mathrm{~cm}$, and a gain between 50 and $64 \mathrm{~dB}$. The settings were adjusted for each subject to produce the clearest images of the analyzed muscles. The same experienced examiner $(\sim 120 \mathrm{~h}$ of training and 600 images acquired before of the study) conducted the acquisition of two images for each of the given femur percentage lengths $(40,50,60$, and $70 \%)$. For the acquisition procedure, the probe was placed transversely in parallel to the intercondylar line using a coupled guide on the subject's thigh (probe guide) (Figure 1C). This procedure was performed with constant speed (controlled by a metronome) and lasted between 12 and $15 \mathrm{~s}$ depending on the subject's thigh circumference. Sixteen images per subject were obtained for the rectus femoris and vastus lateralis muscle CSA analysis ( 8 pre-test +8 post-test). Following the acquisition procedure, CSAs of each muscle scan were manually demarcated by a blinded examiner using specific software (OsiriX MD 6.0, Bernex, Switzerland) (Figure 2). For the data analysis, the rectus femoris and vastus lateralis muscle CSA mean values were calculated using two images acquired at each percentage of the femur length. Finally, based on the 40, 50, 60, and 70\% length 
236

237

238

239

240

241

242

243

244

245

246

247

248

249

250

251

252

253

254

255

256

257

258

259

260

261

262

263

264

265

266

267

268

269

270

271

272

273

274

measurements, the sum of four CSAs of each analyzed muscle was calculated, generating a summary CSA value per muscle to avoid a possible misinterpretation based on one measurement site only (Noorkoiv et al., 2010). This value was used in the statistical data analysis.

\section{- PLEASE INSERT FIGURE 2 HERE -}

\section{Experimental Sessions 3, 4 and 41 (strength tests)}

The strength tests were conducted during the third session in order to familiarize the subjects with the procedures to be performed during the following session. After positioning the participants in the equipment, a familiarization MVIC test was conducted encompassing two attempts of $5 \mathrm{~s}$ in duration with knee flexion angles of $30^{\circ}$ and $90^{\circ}$ (knee extended $=0^{\circ}$ ). MVIC tests were conducted with both legs with 2-minute rest periods between each attempt (Lacerda et al., 2019). The testing order was randomized between legs. The same order was maintained during the post-test session. The highest force value registered for each attempt at knee flexion angles of $30^{\circ}$ and $90^{\circ}$ was used in further data analyses. During the MVIC test, a verbal command was given on which the subject exerted a maximum force against the fixed lever of the knee extensor machine. Visual feedback of the force trace was provided to the subject as well as verbal instruction from the examiners to achieve maximum strength. The load cell raw data (Tedea, Bavaria, Germany) were converted into digital data (Biovision, Wehrheim, Germany) and filtered through a $4^{\text {th }}$-order Butterworth low-pass filter with a cutoff frequency of $10 \mathrm{~Hz}$.

The 1RM test familiarization was performed 10 minutes after the completion of the MVIC test. Initially, according to procedures described by Lacerda et al. (2016, 2019), subjects performed 10 repetitions without any weight on the equipment. The 1RM was determined in concentric mode within a maximum of 6 attempts with 5-minutes rest periods in between (Lacerda et al., 2016, 2019). In addition, a 5-minute rest period was given between the tests conducted with each of the lower limbs.

In session 4, the MVIC and 1RM tests of the familiarization session were repeated. These tests were also repeated in the $41^{\text {st }}$ experimental session with a rest interval of at least $48 \mathrm{~h}$ following the previous session 40 (ultrasound measurements). The data measured in sessions 4 and 41 were used for statistical analysis.

Experimental Sessions 5 to 39 (training period)

After the initial testing period, the 14-week training commenced (35 training sessions). All participants completed $100 \%$ of the training sessions. The experimental protocols consisted of 34 sets at 50-60\% 1RM with 3-minute rest periods in between. In the 2-s RD protocol, subjects completed each repetition in 2 seconds ( 1 second concentric, 1 second eccentric). In the 6-s RD protocol, subjects perform each repetition in 6 seconds (3 seconds concentric, 3 seconds eccentric). The protocols complied with recommendations for resistance training and muscle hypertrophy (ACSM, 2009). Previously, training protocols with similar concentric and eccentric durations were already investigated in our laboratory or in others' (Lacerda et al., 2016, 2019; Sakamoto \& Sinclair, 2012). For both protocols, all sets were executed until the subjects were unable to complete the concentric action within the required $\operatorname{ROM}\left(70^{\circ}\right)$. 
275

276

277

278

279

280

281

282

283

284

285

286

287

288

289

290

291

292

293

294

295

296

297

298

299

300

301

302

303

304

305

306

307

308

309

310

311

312

313

During the first two weeks, training sessions included 3 sets at $50 \%$ of 1 RM. At third week ( $6^{\text {th }}$ training session), the intensity was increased to $60 \%$ of 1 RM. From week $9\left(20^{\text {th }}\right.$ training session) until the end of the training period, one more set was added such that the subjects. Given that any variation of the load characteristics in addition to the RD could possibly bias the training adaptations, the present study controlled the load configuration and progression.

Every two weeks, beginning in the third week ( $6^{\text {th }}$ training session $), 1 R M$ tests for both legs were re-assessed on a weekly basis before the first training session. A 10-minute rest period between the 1RM test and the start of the training session was provided. During these sessions, the 1RM test was conducted at the same day time as in the pre-test to standardize the circadian rhythm, which may possibly influence strength performance.

Experimental Sessions 6 and 39 ( $2^{\text {nd }}$ and $35^{\text {th }}$ training sessions) (force and electromyography measurements)

The surface EMG procedure (Biovision, Wehrheim, Germany) followed the recommendations by Hermens et al. (2000). For the rectus femoris and vastus lateralis muscles, bipolar surface electrodes ( $\mathrm{Ag} / \mathrm{AgCl}$ - 3M-2223, Brazil) were aligned parallel to the muscle fiber orientation. Prior to the electrode placement above the muscle bellies, the skin areas were shaved, cleaned with alcohol using a cotton pad. The inter-electrode distance was $4 \mathrm{~cm}$ which each electrode to be placed $2 \mathrm{~cm}$ distant from the center of the muscle belly. The ground electrode was attached above the patella. After the electrode attachment, a silk paper was used to assess their positions as well as the patella and other relevant points on the skin. In addition, the subject's two thighs were photographed with the electrodes positioned. These procedures were conducted in the $6^{\text {th }}$ session to map the electrode positions on the thigh and to verify high reproducibility in the post-test measurements (39 th session).

To measure the ROM and the muscle action durations during both protocols, the angular displacement was recorded using a potentiometer (aligned with volunteer's knee-joint). For all training sessions, this device was coupled to the rotational axis of the knee extension device. The potentiometer raw data were converted into angular displacement data and filtered through a $4^{\text {th }}$ order Butterworth low-pass filter with a cutoff frequency of $10 \mathrm{~Hz}$. The duration of each muscle action was comprised of the time between the maximum $\left(100^{\circ}\right.$ of knee flexion) and minimum ( $30^{\circ}$ of knee flexion) angular positions. Thus, the duration of the concentric action corresponded to the period between the maximum and minimum angular positions. In turn, the duration of the eccentric action corresponded to the time between the minimum and maximum angular positions. Additionally, concentric/eccentric durations and the RDs were determined throughout the angular displacement time. This potentiometer data provided online information on a laptop screen for the subjects regarding the duration and ROM data of each muscle action throughout the training sessions and tests (Lacerda et al., 2016, 2019). Moreover, a metronome was used to help subjects maintain pre-established RDs.

All electromyographic, load cell, and potentiometer signals were synchronized and converted by an A/D board (Biovision, Wehrheim, Germany) with a sampling rate of 4,000 Hz. 
314 DasyLab software (Version. 11.0; Measurement Computing Corporation, Massachusetts, USA)

315

316

317

318

319

320

321

322

323

324

325

326

327

328

329

330

331

332

333

334

335

336

337

338

339

340

341

342

343

344

345

346

347

348

349

350

351

352

was used to record and process the data. The methodological procedures to record force measurements were detailed in the strength tests section. The electromyographic data acquisition was amplified (factor 500) and filtered ( $4^{\text {th }}$-order Butterworth band-pass filter of 20$500 \mathrm{~Hz}$ ) to calculate the EMG amplitude as the root mean square. Before commencing each experimental session $\left(6^{\text {th }}\right.$ or $\left.39^{\text {th }}\right)$, subjects were asked to perform a MVIC test for $5 \mathrm{~s}$ on the knee extension machine exercise at $60^{\circ}$ knee flexion (controlled by the potentiometer). The highest force and EMG amplitude values in the MVIC test were used as a reference for the normalization of the subsequent measurements in the exercise protocols. The EMG amplitude during the MVIC was measured over a $1 \mathrm{~s}$ period from $500 \mathrm{~ms}$ before the MVIC peak force to $500 \mathrm{~ms}$ after (Piitulainen et al., 2013). The mean force and EMG amplitude of the concentric muscle actions for each $10^{\circ} \mathrm{knee}$ flexion area $\left(100^{\circ}-90^{\circ}, 90^{\circ}-80^{\circ}\right.$, up to $\left.40-30^{\circ}\right)$ was calculated and normalized by the reference values from the normalization test. As a result, relatives force and EMG amplitude $\mathrm{x}$ knee-joint angle curves (normalized force and EMG-angle) were assessed. This procedure was performed for each protocol. For the acquisition of the force and EMG amplitude values during experimental sessions 6 and 39, participants performed 3 sets with $50 \%$ of the previous $1 \mathrm{RM}$ value in each protocol.

\section{Statistical analyses}

Statistical analysis was performed with SPSS for Windows version 20.0 (SPSS, Inc., Illinois, USA). The normal distribution was verified by the Shapiro-Wilk test. All data were expressed as mean $\pm S D$. For the estimation of effect sizes, eta squared $\left(\eta^{2}\right)$ values are considered to reflect the magnitude of the differences (effect size) in each treatment with values $\leq 0.010$ expressing a trivial effect; values between 0.010 and 0.059 expressing a small effect; values between 0.060 and 0.139 a moderate effect, and values $\geq 0.140$ a large effect.

Initially, paired sample t-tests were used to test for differences between the training protocols in baseline values for the main variables analyzed (CSA, 1RM and MVIC), as well as for EMG amplitude and force values obtained during the normalization. The differences between the training protocols in the CSA scores were analyzed through a two-way repeated-measures analysis of variance test (ANOVA) for each muscle separately, having protocol (2-s RD or 6-s RD) and time (pre and post-test) as factors. In case of significant F-values a Bonferroni adjustment was used for comparison purposes. The intra-rater reliability was verified by the intraclass correlation coefficient $\left(\mathrm{ICC}_{[3,1]}\right)$. For the ICC calculations were conducted for both CSA measures (rectus femoris and vastus lateralis) and for both the test sessions (pre and posttest).

Similar to CSA measurements, a two-way repeated-measures ANOVA test (protocol $\mathrm{x}$ time) was applied for MVIC at $30^{\circ}$ of knee flexion scores. However, the $1 \mathrm{RM}$ and MVIC at $90^{\circ}$ of knee flexion values were significantly different at baseline. Therefore, the baseline values were considered as a covariate, and an analysis of covariance (ANCOVA) was implemented using a within-subject factors model. In addition, the ICC intersession values for 1RM and MVIC were

Peer] reviewing PDF | (2020:03:47292:3:1:NEW 22 Dec 2020) 
353

354

355

356

357

358

359

360

361

362

363

364

365

366

367

368

369

370

371

372

373

374

375

376

377

378

379

380

381

382

383

384

385

386

387

388

389

390

obtained from measures during the third (familiarization) and fourth (pre-test) sessions. The familiarization and pre-test sessions were separated by at least $48 \mathrm{~h}$.

Normalized EMG amplitude-angle relationships for the rectus femoris and the vastus lateralis muscles were established during the $6^{\text {th }}$ and $39^{\text {th }}$ sessions to compare EMG amplitude differences between the 2-s and the 6-s RD protocols. A three-way repeated measures ANOVA test (session $\mathrm{x}$ protocol $\mathrm{x}$ knee joint angle) was conducted to analyze the training effects in the normalized EMG amplitude for each muscle. Similar to EMG amplitude responses, a three-way repeated measures ANOVA test (session x protocol $\mathrm{x}$ knee joint angle) was used to compare normalized force-angle relationships in the $6^{\text {th }}$ and $39^{\text {th }}$ sessions. When necessary, a post hoc Bonferroni honest significant difference test was used to identify the differences reported in the ANOVA's. Furthermore, the EMG amplitude and force values for each protocol obtained during the normalization test from experimental sessions 6 and 39 were compared by t-test. This procedure aimed to identify possible differences in measurements in both lower limbs of the same individual. Thus, the feasibility of comparing the EMG amplitude and force responses of the two training protocols should be established.

In addition, paired sample t-tests were used to compare the RDs, ROM and TUT mean values for all sets during training sessions between investigated protocols. Finally, given the number of repetitions for each protocol does not meet the precepts for a parametric analysis, Wilcoxon test was used to compare the values in this variable for both protocols. This data is presented as median (number repetitions per set) and interquartile interval values. The level of the error probability/statistical significance was set at $\mathrm{p} \leq 0.05$ for all statistical tests.

\section{Results}

CSA

The intra-rater reliability values found in these sessions were 0.99 for both analyzed muscles. No significant interaction was observed between protocol and time for the rectus femoris CSA ( $\mathrm{F}_{1,9}$ $=1.889 ; p=0.203 ; \eta^{2}<0.010$ "trivial"). Also, no significant differences between groups were found (main protocol effect; $\mathrm{F}_{1,9}=0.001 ; p=0.972 ; \eta^{2}<0.010$ "trivial"). Both training protocols showed significant increases in rectus femoris CSA after training period (main time effect; $F_{1,9}=$ 64.353; $p<0.001 ; \eta^{2}=0,821$ "large") (Figure 3A).

In addition, no significant interaction was observed between protocol and time for the vastus lateralis CSA ( $\mathrm{F}_{1,9}=0.867 ; p<0.376 ; \eta^{2}<0.010$ “trivial”). No significant difference between the 2-s and the 6-s RD protocols were found (main protocol effect; $\mathrm{F}_{1,9}=0.009 ; p=$ $0.928 ; \eta^{2}<0.010$ "trivial”). Both training protocols showed significant increases in vastus lateralis CSA after training period (main time effect; $F_{1,9}=50.664 ; p<0.001 ; \eta^{2}=0,814$ "large") (Figure 3B). 
391

392

393

394

395

396

397

398

399

400

401

402

403

404

405

406

407

408

409

410

411

412

413

414

415

416

417

418

419

420

421

422

423

424

425

1RM

The ICC intersession value for 1RM tests was 0.98. There were significant difference in 1RM values at baseline (Pre; $t_{9}=2.688 ; p=0.025$ ). When baseline differences in 1RM values were taken into account (within-subject factors ANCOVA), no significant main protocol effect was found $\left(\mathrm{F}_{1,9}=0.412 ; p=0.820 ; \eta^{2}<0.010\right.$ "trivial") (Figure 4$)$.

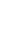

MVIC at $30^{\circ}$ of knee flexion

The ICC intersession value for MVIC test at $30^{\circ}$ of knee flexion was 0.96 . A significant interaction was observed between protocol and time for MVIC test at $30^{\circ}$ of knee flexion measurements $\left(F_{1,9}=6.576 ; p=0.030 ; \eta^{2}=0,049\right.$ “small"), with higher values for the 6-s RD protocol. Furthermore, a significant main effect of time $\left(F_{1,9}=7.581 ; p=0.023 ; \eta^{2}=0,273\right.$ "large") was detected. Conversely, no significant main effect of protocol was found $\left(F_{1,9}=1.990\right.$; $p=0.192 ; \eta^{2}=0,101$ "moderate") (Figure 5).

- PLEASE INSERT FIGURE 5 HERE -

MVIC at $90^{\circ}$ of knee flexion

The ICC intersession value for MVIC test at $90^{\circ}$ of knee flexion was 0.94 . There were significant differences at baseline (Pre; $t_{9}=2.640 ; p=0.027$ ). When baseline differences in MVIC test at $90^{\circ}$ of knee flexion values were taken into account (within-subject factors ANCOVA), no significant main protocol effect was found $\left(\mathrm{F}_{1,9}<0.001 ; p=0.996 ; \eta^{2}<0.010\right.$ "trivial") (Figure $6)$.

\section{- PLEASE INSERT FIGURE 6 HERE -}

Normalized EMG amplitude-angle relationship

The EMG amplitude values obtained during the normalization tests showed no significant difference between the 2-s RD and the 6-s RD training protocols (rectus femoris $-t_{19}=0.701, p=$ 0.503 ) (vastus lateralis $-t_{19}=0.773, p=0.455$ ).

No significant interaction between time $\mathrm{x}$ protocol $\mathrm{x}$ knee joint angle was observed for the normalized EMG amplitude data of rectus femoris $\left(F_{6,54}=2.025 ; p=0.143 ; \eta^{2}<0.010\right.$ "trivial") and vastus lateralis muscles $\left(F_{6,54}=2.640 ; p=0.106 ; \eta^{2}<0.010\right.$ "trivial"). However, significant differences in the rectus femoris EMG amplitude were observed between the 2-s RD and the 6-s RD training protocols in all knee-joint angles analyzed during 6th and 39th sessions (protocol x knee-joint angle interaction $-\mathrm{F}_{6,54}=66.554 ; p<0.001 ; \eta^{2}=0.199$ "large") (Figure 7AB). The 2-s RD protocol resulted in larger normalized EMG amplitude scores in six of the seven knee-joint angles analyzed $\left(100-90^{\circ}\right.$ to $\left.50-40^{\circ}\right)$. Conversely, the 6-s RD protocol provided significantly larger rectus femoris EMG amplitude in the last knee-joint angle (40-30o) only. The 
426

427

428

429

430

431

432

433

434

435

436

437

438

439

440

441

442

443

444

445

446

447

448

449

450

451

452

453

454

455

456

457

458

459

460

same results were verified for the vastus lateralis EMG amplitude (protocol $\mathrm{x}$ knee joint angle interaction $-F_{6,54}=51.007 ; p<0.001 ; \eta^{2}=0.179$ "large") (Figure 8AB).

In addition, no significant interactions were detected between time $\mathrm{x}$ knee joint angle for rectus femoris $\left(F_{6,54}=2.643 ; p=0.070 ; \eta^{2}<0.010\right.$ "trivial") $)$ and vastus lateralis muscles $\left(F_{6,54}=\right.$ $0.414 ; p=0.866 ; \eta^{2}<0.010$ "trivial"). Also, no significant interactions were detected between time $\mathrm{x}$ protocol for rectus femoris $\left(F_{1,9}=0.168 ; p=0.692 ; \eta^{2}<0.001\right.$ "trivial") and vastus lateralis muscles $\left(F_{1,9}=0.014 ; p=0.910 ; \eta^{2}<0.001\right.$ "trivial"). No significant main effect for the time factor (rectus femoris: $\mathrm{F}_{1,9}=0.143 ; p=0.714 ; \eta^{2}<0.010$ "trivial") (vastus lateralis: $\mathrm{F}_{1,9}=$ 2.434; $p=0.153 ; \eta^{2}=0.043$ "small”) was detected. In contrast, significant main effects were found for the training protocol (rectus femoris: $F_{1,9}=29.46 ; p<0.001 ; \eta^{2}=0.202$ "large") (vastus lateralis: $F_{1,9}=16.131 ; p=0.003 ; \eta^{2}=0.225$ "large") and for knee-joint angle (rectus femoris: $F_{6,54}=15.673 ; \mathrm{p}<0.001 ; \eta^{2}=0.111$ "large") (vastus lateralis: $F_{6,54}=10.179 ; p>0.001$; $\eta^{2}=0.037$ “small”).

\section{Normalized force-angle relationship}

The force values obtained during the normalization showed no significant difference between both training protocols analyzed $\left(t_{19}=0.732 ; p=0.478\right)$.

Significant differences in the normalized force-angle relationship were observed between the 2-s RD and the 6-s RD training protocols during $6^{\text {th }}$ and $39^{\text {th }}$ sessions (protocol $x$ knee-joint angle $x$ time $-F_{6,54}=2.652 ; p=0.025 ; \eta^{2}=0.033$ “small”). In the $6^{\text {th }}$ session, the $2-\mathrm{s}$ RD protocol exhibited significantly larger normalized force values in the first four knee-joint angles analyzed $\left(100-90^{\circ}\right.$ to $\left.70-60^{\circ}\right)$. The same was true for the first three knee-joint angles $\left(100-90^{\circ}\right.$ to $\left.80-70^{\circ}\right)$ in the $39^{\text {th }}$ experimental session. In contrast, for the 6-s RD protocol larger normalized force values were found in the last two knee-joint angles (50-40 40-30 $)$ during sessions 6 and 39. In addition, significant changes in the force $\mathrm{x}$ angle relationships between training sessions were detected only for the 2-s RD protocol. These changes were related to a reduction in the force values from $70-60^{\circ}$ until $40-30^{\circ}$ of knee flexion (Figure $9 \mathrm{AB}$ ).

Moreover, significant interactions were found between the time $\mathrm{x}$ knee joint angle $\left(F_{6,54}=\right.$ $14.243 ; p<0.001 ; \eta^{2}=0.011$ "small”') and the protocol $\mathrm{x}$ knee-joint angle $\left(F_{1,9}=296.591 ; p<\right.$ $0.001 ; \eta^{2}=0.370$ "large"). No significant interaction was observed for the interaction of time $\mathrm{x}$ protocol $\left(F_{1,9}=2.483 ; p=0.150 ; \eta^{2}<0.010\right.$ "trivial"). Finally, no significant main effect for time $\left(F_{1,9}=1.807 ; p=0.212 ; \eta^{2}=0.074\right.$ "moderate" $)$ and for protocol $\left(F_{1,9}=1.555 ; p=0.703 ; \eta^{2}\right.$ $<0.010$ "trivial"), but a significant main effect for knee-joint angle $\left(F_{6,54}=7.453 ; p<0.001 ; \eta^{2}=\right.$ 0.026 "small") were identified.

- PLEASE INSERT FIGURE 9 HERE - 
461

462

463

464

465

466

467

468

469

470

471

472

473

474

475

476

477

478

479

480

481

482

483

484

485

486

487

488

489

490

491

492

493

494

495

496

497

498

499

500

\section{RD, TUT, Number of repetitions and ROM}

As expected, 6-s RD protocol showed longer average RD than 2-s RD protocol $(2.04 \pm 0.08 \mathrm{~s}$; $5.98 \pm 0.09 \mathrm{~s}$, respectively; $\left.t_{69}=284.488, p<0.001\right)$. In addition, larger TUT mean values were observed in the 6-s RD protocol (mean for all sets $=43.47 \pm 10.92 ; 1^{\text {st }}$ set $=52.5 \pm 15.83,2^{\text {nd }}$ set $=42.58 \pm 12.25$, last set $\left(3^{\text {rd }}\right.$ or $\left.\left.4^{\text {th }}\right)=36.98 \pm 10.89\right)$ as compared to the $2-\mathrm{s}$ RD protocol (mean for all sets $=30.51 \pm 7.52 ; 1^{\text {st }}$ set $=38.1 \pm 10.55,2^{\text {nd }}$ set $=29.57 \pm 7.98$, last set $\left(3^{\text {rd }}\right.$ or $\left.4^{\text {th }}\right)=$ $25.09 \pm 7.17)\left(t_{69}=15.951 ; p<0.001\right)$. In regard to the number of repetitions, the Wilcoxon test showed significantly larger median values for the 2-s RD protocol (median for all sets $=14$ [12$17] ; 1^{\text {st }}$ set $=18$ [21.25-17], $2^{\text {nd }}$ set $=14.5$ [16.25-12.75], last set $\left(3^{\text {rd }}\right.$ or $\left.4^{\text {th }}\right)=12$ [14-11] $)$ as compared to the 6-s RD protocol (median for all sets $=7[6-8] ; 1^{\text {st }}$ set $=9[8-10], 2^{\text {nd }}$ set $=7[6-8]$, last set $\left(3^{\text {rd }}\right.$ or $\left.\left.4^{\text {th }}\right)=6[5-7]\right)\left(U_{69}=7.294 ; p<0.001\right)$. Last not least, but no significant differences were detected between the 2-s RD and the 6-s RD protocols in the ROM average values (2-s RD: $70.77 \pm 0.79^{\circ}$; 6-s $\mathrm{RD}: 70.99 \pm 0.65^{\circ} ; t_{69^{\prime}}=1.903, p=0.06$ ).

\section{Discussion}

The purpose of this study was to compare the strength and muscle hypertrophy responses induced by two protocols with different RDs performed to MF. In addition, we aimed to verify the effects of these RD strategies on knee extension force and EMG amplitude in the rectus femoris and vastus lateralis muscles. To the best of our knowledge, no other studies have compared these chronic adaptations for resistance training with different RD to MF by matching intensity, set, and rest and, in addition to that, using ultrasound measurements from four different parts of the muscle. The main results of the present study were: 1) the 2-s RD protocol showed similar effects in maximum dynamic strength and muscle hypertrophy compared to the 6-s protocol; 2) the 6-s RD protocol induced larger gains in MVIC at knee flexion of $30^{\circ}$ than the 2-s RD. However, both protocols induced similar increases in MVIC at for the $90^{\circ}$ of knee angle and in 1RM performance. Thus, RD appears to influence the joint-angle specific strength gains only in shorter muscle lengths.

Both rectus femoris and vastus lateralis muscles had a similar increase in muscle CSA for the 2-s RD and the 6-s RD protocols after 35 training sessions with a trivial effect size $\left(\eta^{2}<\right.$ 0.010). Different factors have shown to explain the increase in muscle hypertrophy: elevated EMG amplitude (e.g. higher recruitment of motor units and/or firing rate) (Hunter et al., 2004; Lacerda et al., 2016), increase in force production (Sampson \& Groeller, 2016; Sampson et al., 2014), and also differences in the training volume and TUT (Schoenfeld \& Grgic, 2019). In general, a higher training volume is connected to an increase in the TUT. For instance, studies investigating resistance protocols balanced training volume, but more substantial muscle hypertrophy after training was detected with longer RD and TUT (Tanimoto \& Ishii, 2006). Nevertheless, in the present study, the 6-s RD protocol was executed with average TUTs approximately $25 \%$ larger than in the $2-\mathrm{s}$ RD protocol (43 s vs. $30 \mathrm{~s}$, respectively), while the 2-s $\mathrm{RD}$ protocol encompassed double of the 6-s RD training volume (14 vs. 7 repetition per set, respectively). Additionally, the present study also showed higher EMG amplitude for the 2-s 
501 compared to 6-s RD protocol, in line with previous findings (Lacerda et al., 2016; Sakamoto \& 502 Sinclair, 2012), which may also have contributed to muscle hypertrophy. Thus, the larger 503 training volume and higher EMG amplitude in the 2-s RD protocol, and the longer TUT in the 6504 s RD protocol, may have counterbalanced each other and led to similar impact on muscle 505 hypertrophy, explaining the present findings. Therefore, these results suggest that protocols with 506 shorter RD performed with a higher training volume and higher EMG amplitude could lead to 507 muscle hypertrophy, to the same extent as protocols with longer RD performed with longer TUT.

The increase in the number of motor units recruited during resistance training has been

510

511

512

513

514

515

516

517

518

519

520

521

522

523

524

525

526

527

528

529

530

531

532

533

534

535

536

537

538

539

540 pointed out as a central factor to trigger muscle hypertrophy (Schoenfeld, 2013). The present study demonstrated a higher EMG amplitude for the 2-s RD as compared to the 6-s RD protocol during most of the ROM measurements conducted $\left(100^{\circ}\right.$ to $\left.40^{\circ}\right)$. In contrast, the 6-s RD protocol showed higher EMG amplitude only in the last $10^{\circ}$ range for concentric ROM ( $40^{\circ}$ to $\left.30^{\circ}\right)$. This result suggests an increased motor unit recruitment during most concentric ROM for the 2-s RD. However, these differences may not have been sufficient to result in a marked increase in CSA to the 2-s RD protocol as compared to the 6-s RD. Although increased EMG amplitude is associated with higher motor unit recruitment other factors may contribute to the changes in the EMG amplitude as well such as increased firing frequency and synchronization of motor units (Hunter et al., 2004). Therefore, care must be taken when interpreting EMG data obtained prior and after the resistance training period.

According to EMG amplitude, higher force values were found during the 2-s RD protocol at the beginning of concentric ROM while higher force values were observed for 6-s RD protocol at the end of concentric ROM. Moreover, at the $39^{\text {th }}$ experimental session, the 2-s RD protocol was executed with higher muscle forces $\left(100^{\circ}\right.$ to $\left.70^{\circ}\right)$ during most ROM areas compared to the 6-s RD protocol $\left(50^{\circ}\right.$ to $\left.30^{\circ}\right)$. These results agree with previous results showing higher muscle forces at the beginning of concentric ROM when performing faster movements (Sampson et al., 2014). It should be noted, however, that, although the RD of $2 \mathrm{~s}$ was only a third of the 6-s $\mathrm{RD}$, the magnitude of the forces applied in the two experimental situations were similar (43\% of MVIC at $39^{\text {th }}$ experimental session). This result does not agree with previous studies (Sampson et al., 2014). Sampson et al. (2014) compared protocols with different RDs and showed differences in force produced close to $25 \%$ at the onset of concentric action. However, their participants were instructed to perform ballistic movements or controlled movements within $4 \mathrm{~s}$. With similar protocols to those in the last study, Sampson and Groeller (2016) found similar gains in muscle hypertrophy in both experimental conditions after 12 weeks of training. For these authors, the higher force applied during ballistic movements would be a determinant factor for muscle hypertrophy. Therefore, it is possible that in the present study, despite the higher force demand during 2-s RD protocol at the beginning of concentric ROM, similar in force produced between both protocols was essential so that no significant differences could be detected in the CSA data of rectus femoris and vastus lateralis.

In agreement with the CSA overall responses, the average maximal dynamic strength performance (1RM test) was similar between the 2-s RD and the 6-s RD protocols (trivial effect 
541 size, $\left.\eta^{2}<0.010\right)$. As a mixed result, higher force values were detected in the 2-s RD as compared 542 to the 6-s RD protocol during the initial phase of the ROM in the concentric action while the 543 1RM gains were similar between protocols. This outcome did not confirm the trend of superior 544 performance when training with fast movement velocities and moderate intensities (60-79\% 545 1RM), as was indicated in a previous meta-analysis (Davies et al., 2017). Hence, our initial 546 hypothesis was rejected.

547 In line with previous research (Sampson \& Groeller, 2016), we did not find differences 548 in 1RM performance originating from different RD protocols using the same load intensity. In 549 contrast, other investigators had found higher 1RM gains when utilizing fast movement 550 velocities (González-Badillo et al., 2014; Padulo et al., 2012). The discrepancy between these 551 results may be associated to different RD adopted in these studies. Although the movement time 552 in the 2-s RD protocol was three times shorter than in the 6-s RD protocol, participants were not 553 instructed to perform explosive movements, which was the case in other studies (González554 Badillo et al., 2014; Padulo et al., 2012). It has been reported a greater EMG amplitude and 555 impulse production when ballistic movements were performed (Maffiuletti et al., 2016). This 556 factor may have influenced the occurrence of adaptations favorable to the 1RM increase for 557 faster protocols in these studies (González-Badillo et al., 2014; Padulo et al., 2012), which was 558 not observed in the present research. Importantly, in the abovementioned studies at least one of 559 the analyzed protocols was performed to MF. Thus, differences within the levels of effort must 560 be assumed (González-Badillo et al., 2014; Padulo et al., 2012; Sampson \& Groeller, 2016).

561 Moreover, performing repetitions to MF provide a maximum effort for all individuals during 562 both protocols (Dankel et al., 2017), which may have been a determinant factor in not having 563 difference in 1RM gains between the two protocols investigated in the present study. Therefore, 564 training to MF could hamper the effect of RD on maximum dynamic strength performance 565 observed in previous studies.

In addition, it has been reported that exposure to successive 1RM tests on a three-week 568 protocols (Morton et al., 2016). Consecutive measurements may evolve to a similar motor 569 pattern within the 1RM tests. Hence, it appears possible that existing differences between 570 training protocols may not be detected (Bernardi et al., 1996). Data from an untrained control 571 limb would enable a better understanding of the effect of repeated exposure of both trained limb 572 to 1RM tests. Thus, the lack of a control limb was a limitation of the present study and must be 573 considered when interpreting the results. Accordingly, studies on RD effects showing 574 inconclusive results for the 1RM tests may have other outcomes further to the 1RM measurement 575 procedures (Pereira \& Gomes, 2007). Therefore, MVIC tests performed at different joint angles 576 may be a valid alternative to investigate the effect of different training protocols on muscle 577 strength responses. Given that protocols with different RD provide changes in force production 578 that varies over the ROM (Sampson et al., 2014), it was hypothesized that greater isometric 579 maximal strength gains would be found in knee-joint angles with higher instantaneous force 580 values applied. 
581

582

583

584

585

586

587

588

589

590

591

592

593

594

595

596

597

598

599

600

601

602

603

604

605

606

607

608

609

610

611

612

613

614

615

616

617

618

619

620

Similar to the 1RM scores, the increases in the MVIC values at $90^{\circ}$ of knee flexion did show comparable improvements by both RD training protocols. A trivial effect size $\left(\eta^{2}<0.010\right)$ reinforces the result found by t-tests. Based on the TE values, the individual analyses showed that $40 \%$ of the sample did not respond substantially differently for either of the two training protocols. In turn, 3 participants each showed substantial improvements in either the 2-s RD or the 6-s RD protocol. Conversely, larger gains were observed in the MVIC at $30^{\circ}$ of knee flexion for 6-s RD protocol. However, a small effect size was observed for the analysis of the interaction effect between protocol and time $\left(\eta^{2}=0.048\right)$ and must be considered when interpreting the results. Based on results from previous studies (Alegre et al., 2014), we hypothesized that the distinct force-angle relationship obtained during the two training protocols would influence the MVIC gains at different knee-joint angles. These previous studies, in general, presented larger maximum strength gains in the joint angles near the training angle/ROM used in the corresponding training. In contrast, in the present study, our subjects performed both training protocols with the same $\mathrm{ROM}\left(70^{\circ}\right)$ varying the force generated along with the angular exercise range $\left(100^{\circ}\right.$ to $\left.30^{\circ}\right)$. Data from the $39^{\text {th }}$ training session (Figure $\left.8 \mathrm{~B}\right)$ show that in the initial $(100$ $\left.90^{\circ}\right)$ and final $\left(40-30^{\circ}\right)$ ranges of the concentric testing action largest differences in force production were detected between the protocols ( 7 vs. 13\%). Additionally, the 2-s RD protocol showed higher values at the beginning and the 6-s RD protocol at the end of the testing movement. Given that type of mechanical stimulus (i.e. type of contraction) may contribute to the specific training adaptations (Buckthorpe et al., 2015) it appears possible that the higher force production in the 6-s RD protocol compared to the 2-s RD protocol at the end of concentric action might be sufficient to produce differences in isometric force gains at $30^{\circ}$ but not at $90^{\circ}$ of knee flexion. Furthermore, increases in isometric force after training have been associated with increases in EMG amplitude (Noorkoiv et al., 2014). The EMG-angle relationship for the 6-s RD protocol showed higher normalized EMG amplitude at $40-30^{\circ}$ of knee-joint angle (end of concentric action) and so help to explain the force gains at $30^{\circ}$ of knee flexion. Moreover, previous studies verified joint-angle specific strength gains close to trained angle/ROM (Alegre et al., 2014) while others only showed joint-angle specific strength gains for resistance training performed in shorter muscle lengths (Noorkoiv et al., 2014), reinforcing the results obtained in the present study up to $30^{\circ}$ of knee flexion. However, it should be emphasized that the mechanisms suggested explaining distinct strength gains at specific joint angles are still poorly understood (Alegre et al., 2014; Noorkoiv et al., 2014).

A limitation of the intra-individual experimental design is a possible cross-training or cross-education effect (Beyer et al., 2016). There is evidence in the literature indicating that the cross-training effect, if it occurs, would be restricted to neural parameters and muscle strength gains while morphological changes (e.g., CSA) would not be influenced by this effect (Beyer et al., 2016). In this respect, muscle strength gains in the contralateral limb should evolve from an increase in the motor neuron activation and are not related to morphological adaptations. However, previous studies investigating the crossing-effect for EMG amplitude showed inconclusive results (Hortobágyi et al., 1997; Lee \& Carroll, 2007). For example, Hortobágyi et

Peer) reviewing PDF | (2020:03:47292:3:1:NEW 22 Dec 2020) 
621 al. (1997) found that changes in the EMG amplitude of the untrained limb depending on the 622 training mode performed (e.g., type of muscle action). The neuromuscular changes were similar 623 to the changes in muscle strength. In addition, researchers found that the cross-training effect 624 contributes to approximately $7.8 \%$ of the muscle strength gain of the contralateral limb (Munn et 625 al., 2004). Such adaptation was explained by neural mechanisms involving acute facilitation 626 within the motor cortex of the untrained contralateral limb following excitation of the trained 627 limb (Fisher et al., 2016). The training protocols were performed with a minimum interval of $24 \mathrm{~h}$ 628 in order to minimize the acute effect of unilateral training reducing the maximal strength performance in the contralateral limb. Finally, and most important to our study, it has been argued that, when both limbs of an individual are trained with different protocols, the cross-

632

633

634

635

636

637

638

639

640

641

642

643

644

645

646

647

648

649

650

651

652

653

654

655

656

657

658

659

660 training effect is minimal or non-existent (Munn et al., 2004; Bell et al., 2020). Hence, we expected that any difference in the strength responses between limbs would be due to training protocols and not owing to a crossing effect (Fisher et al., 2016).

\section{Conclusions}

This study showed that protocols with different RD performed to MF produced similar muscle hypertrophy gains despite differences in the EMG amplitude and force-angle relationships. Therefore, different training volumes and TUTs based on the different RDs appear to produce a similar stimulus to skeletal muscle growth. Thus, we argue that an increased training volume provided by performing faster movements to MF would promote similar muscle hypertrophy when compared to higher TUTs during slower movements. It is noteworthy that the highly trained individuals possibly require larger training volumes in order to achieve chronic adaptations (i.e. muscle hypertrophy) associated with resistance training as compared to untrained or moderately trained individuals (Figueiredo et al., 2018). Yet, although no differences in 1RM gains between protocols were found, our MVIC data provides important insight for the understanding of joint-angle specific strength responses induced by RDs. We demonstrate that high force production in the end of concentric action during the 6-s RD protocol induced higher maximal isometric strength at $30^{\circ}$ of knee flexion when compared to the 2-s RD protocol.

Repetition duration is considered an essential variable of resistance training (Dankel et al., 2017; Davies et al., 2017; Pereira \& Gomes, 2007; Tanimoto \& Ishii, 2006), but recent studies are not supporting this view on strength and muscle hypertrophy (Davies et al., 2017; Schoenfeld et al., 2015). Nevertheless, this investigation has shown that resistance training performed to MF with longer RD could be a more appropriate strategy to provide greater gains in maximal muscle strength at shortened knee positions, although different RD would induce similar muscle hypertrophy. Thus, the current results have practical applications for individuals seeking health-related improvements in muscular strength and hypertrophy. Overall, it should note that the results presented here are limited to the exercise and subject characteristics similar to those of our current study. However, future studies with females and trained individuals are needed to clarify the impact of protocols with different RDs on the chronic adaptations associated with resistance training performed to MF. 


\section{References}

662 ACSM. (2009). Progression Models in Resistance Training for Healthy Adults. Medicine \& Science in Sports \& Exercise, 41(3), 687-708. https://doi.org/10.1249/MSS.0b013e3181915670

Alegre, L. M., Ferri-Morales, A., Rodriguez-Casares, R., \& Aguado, X. (2014). Effects of isometric training on the knee extensor moment-angle relationship and vastus lateralis muscle architecture. European Journal of Applied Physiology, 114(11), 2437-2446. https://doi.org/10.1007/s00421-014-2967-x

670

Beck, T. (2013). The importance of a priori sample size estimation in strength and conditioning research. Journal of Strength and Conditioning Research, 27(8), 2323-2337. https://doi.org/10.1519/JSC.0b013e318278eea0

Bell, Z. W., Wong, V., Spitz, R. W., Chatakondi, R. N., Viana, R., Abe, T., \& Loenneke, J. P. (2020). The contraction history of the muscle and strength change: lessons learned from unilateral training models. Physiological measurement, 41(1), 01TR01. https://doi.org/10.1088/1361-6579/ab516c

Bernardi, M., Solomonow, M., Nguyen, G., Smith, A., \& Baratta, R. (1996). Motor unit recruitment strategy changes with skill acquisition. European Journal of Applied Physiology and Occupational Physiology, 74(1-2), 52-59.

Beyer, K. S., Fukuda, D. H., Boone, C. H., Wells, A. J., Townsend, J. R., Jajtner, A. R., Gonzalez, A. M., Fragala, M. S., Hoffman, J. R., \& Stout, J. R. (2016). Short-term unilateral resistance training results in cross education of strength without changes in muscle size, activation, or endocrine response. Journal of Strength and Conditioning Research, 30(5), 1213-1223. https://doi.org/10.1519/JSC.0000000000001219

Buckthorpe, M., Erskine, R. M., Fletcher, G., \& Folland, J. P. (2015). Task-specific neural adaptations to isoinertial resistance training. Scandinavian Journal of Medicine \& Science in Sports, 25(5), 640-649. https://doi.org/10.1111/sms.12292

Chaves, T. S., Pires de Campos Biazon, T. M., Marcelino Eder dos Santos, L., \& Libardi, C. A. (2020). Effects of resistance training with controlled versus self-selected repetition duration on muscle mass and strength in untrained men. PeerJ, 8, e8697. https://doi.org/10.7717/peerj.8697

Damas, F., Barcelos, C., Nóbrega, S. R., Ugrinowitsch, C., Lixandrão, M. E., Santos, L. M. E. d., Conceição, M. S., Vechin, F. C., \& Libardi, C. A. (2019). Individual muscle hypertrophy and strength responses to high vs. low resistance training frequencies. Journal of Strength and Conditioning Research, 33(4), 897-901. https://doi.org/10.1519/JSC.0000000000002864

Dankel, S. J., Mattocks, K. T., Jessee, M. B., Buckner, S. L., Mouser, J. G., Counts, B. R., Laurentino, G. C., \& Loenneke, J. P. (2017). Frequency: The Overlooked Resistance Training Variable for Inducing Muscle Hypertrophy? Sports Medicine, 47(5), 799-805. https://doi.org/10.1007/s40279-016-0640-8

Davies, T. B., Kuang, K., Orr, R., Halaki, M., \& Hackett, D. (2017). Effect of Movement Velocity During Resistance Training on Dynamic Muscular Strength: A Systematic Review and Meta-Analysis. Sports Medicine, 47(8), 1603-1617. https://doi.org/10.1007/s40279$\underline{017-0676-4}$

Figueiredo, V. C., de Salles, B. F., \& Trajano, G. S. (2018). Volume for muscle hypertrophy and health outcomes: The most effective variable in resistance training. Sports Medicine, 48(3), 499-505. https://doi.org/10.1007/s40279-017-0793-0 
707

708

709

710

711

712

713

714

715

716

717

718

719

720

721

722

723

724

725

726

727

728

729

730

731

732

733

734

735

736

737

738

739

740

741

742

743

744

745

746

747

748

749

750

751

752

Fisher, J., Blossom, D., \& Steele, J. (2016). A comparison of volume-equated knee extensions to failure, or not to failure, upon rating of perceived exertion and strength adaptations. Applied Physiology, Nutrition, and Metabolism = Physiologie Appliquee, Nutrition et Metabolisme, 41(2), 168-174. https://doi.org/10.1139/apnm-2015-0421

Gehlert, S., Suhr, F., Gutsche, K., Willkomm, L., Kern, J., Jacko, D., Knicker, A., Schiffer, T., Wackerhage, H., \& Bloch, W. (2015). High force development augments skeletal muscle signalling in resistance exercise modes equalized for time under tension. Pflügers Archiv European Journal of Physiology, 467(6), 1343-1356. https://doi.org/10.1007/s00424-0141579-y

González-Badillo, J. J., Rodríguez-Rosell, D., Sánchez-Medina, L., Gorostiaga, E. M., \& ParejaBlanco, F. (2014). Maximal intended velocity training induces greater gains in bench press performance than deliberately slower half-velocity training. European Journal of Sport Science, 14(8), 772-781. https://doi.org/10.1080/17461391.2014.905987

Gonzalez, A. M., Hoffman, J. R., Stout, J. R., Fukuda, D. H., \& Willoughby, D. S. (2016). Intramuscular Anabolic Signaling and Endocrine Response Following Resistance Exercise: Implications for Muscle Hypertrophy. Sports Medicine (Auckland, N.Z.), 46(5), 671-685. https://doi.org/10.1007/s40279-015-0450-4

Hermens, H. J., Freriks, B., Disselhorst-Klug, C., \& Rau, G. (2000). Development of recommendations for SEMG sensors and sensor placement procedures. Journal of Electromyography and Kinesiology: Official Journal of the International Society of Electrophysiological Kinesiology, 10(5), 361-374.

Hortobágyi, T., Lambert, N. J., \& Hill, J. P. (1997). Greater cross education following training with muscle lengthening than shortening. Medicine and Science in Sports and Exercise, 29(1), 107-112.

Hunter, S. K., Duchateau, J., \& Enoka, R. M. (2004). Muscle fatigue and the mechanisms of task failure. Exercise and Sport Sciences Reviews, 32(2), 44-49.

Lacerda, L. T., Marra-Lopes, R. O., Diniz, R. C. R., Lima, F. V., Rodrigues, S. A., MartinsCosta, H. C., Bemben, M. G., \& Chagas, M. H. (2019). Is Performing Repetitions to Failure Less Important Than Volume for Muscle Hypetrophy and Strength? Journal of Strength and Conditioning Research.

Lacerda, L. T., Martins-Costa, H. C., Diniz, R. C. R., Lima, F. V., Andrade, A. G. P., Tourino, F. D., Bemben, M. G., \& Chagas, M. H. (2016). Variations in repetition duration and repetition numbers influence muscular activation and blood lactate response in protocols equalized by time under tension. Journal of Strength and Conditioning Research, 30(1), 251-258. https://doi.org/10.1519/JSC.0000000000001044

Lasevicius, T., Ugrinowitsch, C., Schoenfeld, B. J., Roschel, H., Tavares, L. D., De Souza, E. O., Laurentino, G., \& Tricoli, V. (2018). Effects of different intensities of resistance training with equated volume load on muscle strength and hypertrophy. European Journal of Sport Science, 18(6), 772-780. https://doi.org/10.1080/17461391.2018.1450898

Lee, M., \& Carroll, T. J. (2007). Cross education: possible mechanisms for the contralateral effects of unilateral resistance training. Sports Medicine (Auckland, N.Z.), 37(1), 1-14. https://doi.org/10.2165/00007256-200737010-00001

MacInnis, M. J., McGlory, C., Gibala, M. J., \& Phillips, S. M. (2017). Investigating human skeletal muscle physiology with unilateral exercise models: when one limb is more powerful than two. Applied physiology, nutrition, and metabolism = Physiologie appliquee, nutrition et metabolisme, 42(6), 563-570. https://doi.org/10.1139/apnm-2016-0645 
753

754

755

756

757

758

759

760

761

762

763

764

765

766

767

768

769

770

771

772

773

774

775

776

777

778

779

780

781

782

783

784

785

786

787

788

789

790

791

792

793

794

795

796

797

798

Maffiuletti, N. A., Aagaard, P., Blazevich, A. J., Folland, J., Tillin, N., \& Duchateau, J. (2016). Rate of force development: physiological and methodological considerations. European Journal of Applied Physiology, 116(6), 1091-1116. https://doi.org/10.1007/s00421-016$\underline{3346-6}$

Morton, R. W., Oikawa, S. Y., Wavell, C. G., Mazara, N., McGlory, C., Quadrilatero, J., Baechler, B. L., Baker, S. K., \& Phillips, S. M. (2016). Neither load nor systemic hormones determine resistance training-mediated hypertrophy or strength gains in resistance-trained young men. Journal of Applied Physiology, 121(1), 129-138.

https://doi.org/10.1152/japplphysiol.00154.2016

Munn, J., Herbert, R. D., \& Gandevia, S. C. (2004). Contralateral effects of unilateral resistance training: a meta-analysis. Journal of Applied Physiology, 96(5), 1861-1866. https://doi.org/10.1152/japplphysiol.00541.2003

Noorkoiv, M., Nosaka, K., \& Blazevich, A. J. (2010). Assessment of quadriceps muscle crosssectional area by ultrasound extended-field-of-view imaging. European Journal of Applied Physiology, 109(4), 631-639. https://doi.org/10.1007/s00421-010-1402-1

Noorkoiv, M., Nosaka, K., \& Blazevich, A. J. (2014). Neuromuscular adaptations associated with knee joint angle-specific force change. Medicine \& Science in Sports \& Exercise, 46(8), 1525-1537. https://doi.org/10.1249/MSS.0000000000000269

Padulo, J., Mignogna, P., Mignardi, S., Tonni, F., \& D’Ottavio, S. (2012). Effect of different pushing speeds on bench press. International Journal of Sports Medicine, 33(5), 376-380. https://doi.org/10.1055/s-0031-1299702

Pereira, M. I. R., \& Gomes, P. S. C. (2007). Efeito do treinamento contra-resistência isotônico com duas velocidades de movimento sobre os ganhos de força. Revista Brasileira de Medicina Do Esporte, 13(2), 91-96. https://doi.org/10.1590/S1517-86922007000200005

Piitulainen, H., Botter, A., Merletti, R., \& Avela, J. (2013). Multi-channel electromyography during maximal isometric and dynamic contractions. Journal of Electromyography and Kinesiology, 23(2), 302-310. https://doi.org/10.1016/j.jelekin.2012.10.009

Sakamoto, A., \& Sinclair, P. J. (2012). Muscle activations under varying lifting speeds and intensities during bench press.European Journal of Applied Physiology, 112(3), 1015-1025. https://doi.org/10.1007/s00421-011-2059-0

Sampson, J. A., \& Groeller, H. (2016). Is repetition failure critical for the development of muscle hypertrophy and strength? Scandinavian Journal of Medicine \& Science in Sports, 26(4), 375-383. https://doi.org/10.1111/sms.12445

Sampson, J. A., Donohoe, A., \& Groeller, H. (2014). Effect of concentric and eccentric velocity during heavy-load non-ballistic elbow flexion resistance exercise. Journal of Science and Medicine in Sport, 17(3), 306-311. https://doi.org/10.1016/j.jsams.2013.04.012

Schoenfeld, B. (2013). Potential mechanisms for a role of metabolic stress in hypertrophic adaptations to resistance training. Sports Medicine (Auckland, N.Z.), 43(3), 179-194. https://doi.org/10.1007/s40279-013-0017-1

Schoenfeld, B., \& Grgic, J. (2018). Evidence-Based Guidelines for Resistance Training Volume to Maximize Muscle Hypertrophy. Strength and Conditioning Journal, 40(4), 107-112. https://doi.org/10.1519/SSC.0000000000000363

Schoenfeld, B. J., Contreras, B., Willardson, J. M., Fontana, F., \& Tiryaki-Sonmez, G. (2014). Muscle activation during low- versus high-load resistance training in well-trained men. European Journal of Applied Physiology, 114(12), 2491-2497. https://doi.org/10.1007/s00421-014-2976-9 
799

800

801

802

803

804

805

806

807

808

809

810

811

812

813

814

815
Schoenfeld, B. J., Ogborn, D. I., \& Krieger, J. W. (2015). Effect of Repetition Duration During Resistance Training on Muscle Hypertrophy: A Systematic Review and Meta-Analysis. Sports Medicine, 45(4), 577-585. https://doi.org/10.1007/s40279-015-0304-0

Schoenfeld, B. J., \& Grgic, J. (2019). Does Training to Failure Maximize Muscle Hypertrophy? Strength and Conditioning Journal, 41(5), 108-113. https://doi.org/10.1519/SSC.0000000000000473

Schuenke, M. D., Herman, J. R., Gliders, R. M., Hagerman, F. C., Hikida, R. S., Rana, S. R., Ragg, K. E., \& Staron, R. S. (2012). Early-phase muscular adaptations in response to slowspeed versus traditional resistance-training regimens. European Journal of Applied Physiology, 112(10), 3585-3595. https://doi.org/10.1007/s00421-012-2339-3

Tanimoto, M., \& Ishii, N. (2006). Effects of low-intensity resistance exercise with slow movement and tonic force generation on muscular function in young men. Journal of Applied Physiology, 100(4), 1150-1157. https://doi.org/10.1152/japplphysiol.00741.2005

van den Tillaar, R., Saeterbakken, A. H., \& Ettema, G. (2012). Is the occurrence of the sticking region the result of diminishing potentiation in bench press? Journal of Sports Sciences, 30(6), 591-599. https://doi.org/10.1080/02640414.2012.658844 


\section{Figure 1}

Thigh marking procedures ( $\mathrm{A}$ and $\mathrm{B}$ ) and ultrasound images acquisition (C). Probe guide (indicated by white arrow)(D).
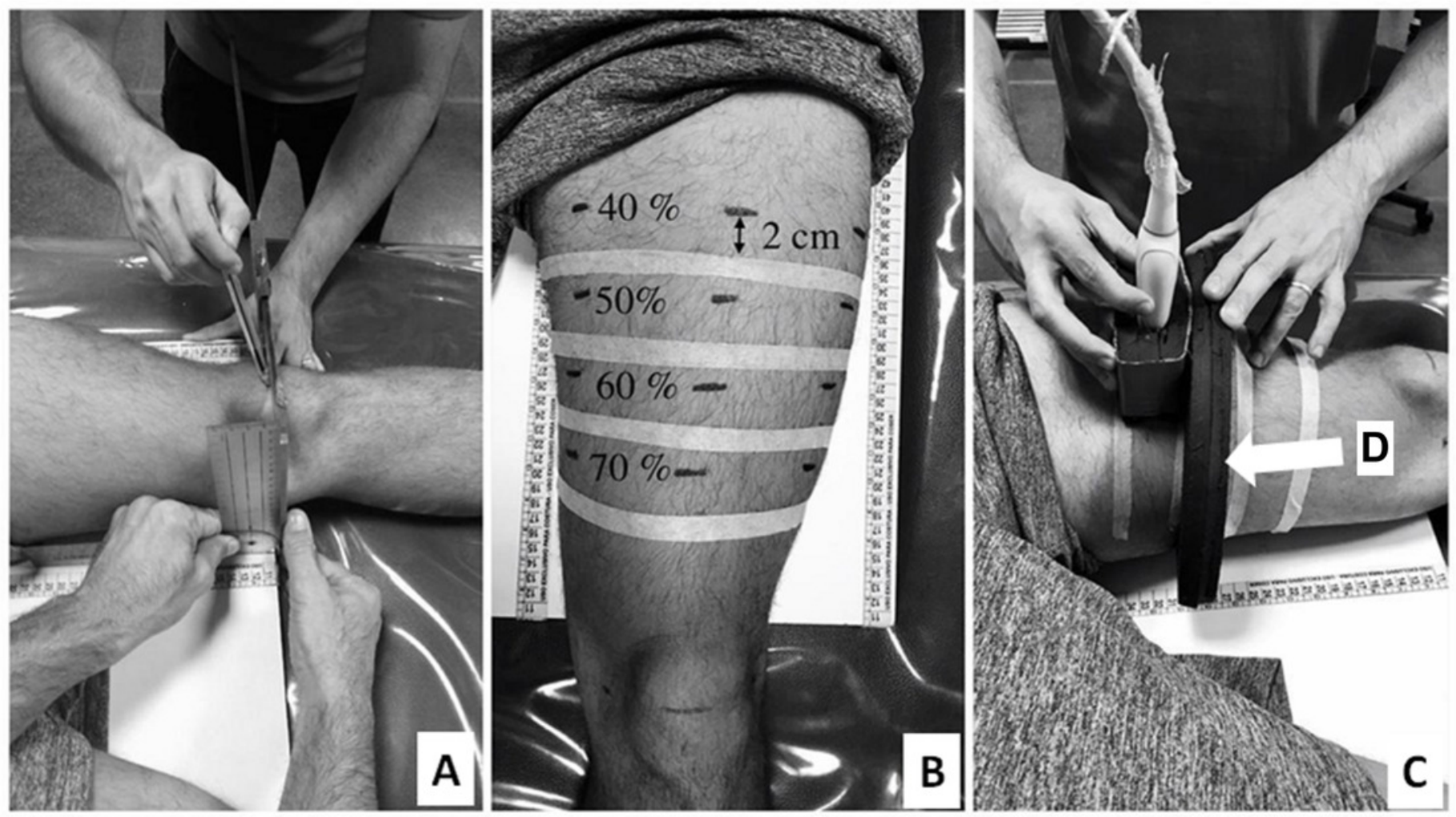
Figure 2

Ultrasound images and cross-sectional areas (CSA) at 40\% (A); 50\% (B), 60\% (C), and $70 \%$ (D) of femur length. Rectus femoris (RF) and vastus lateralis (VL).
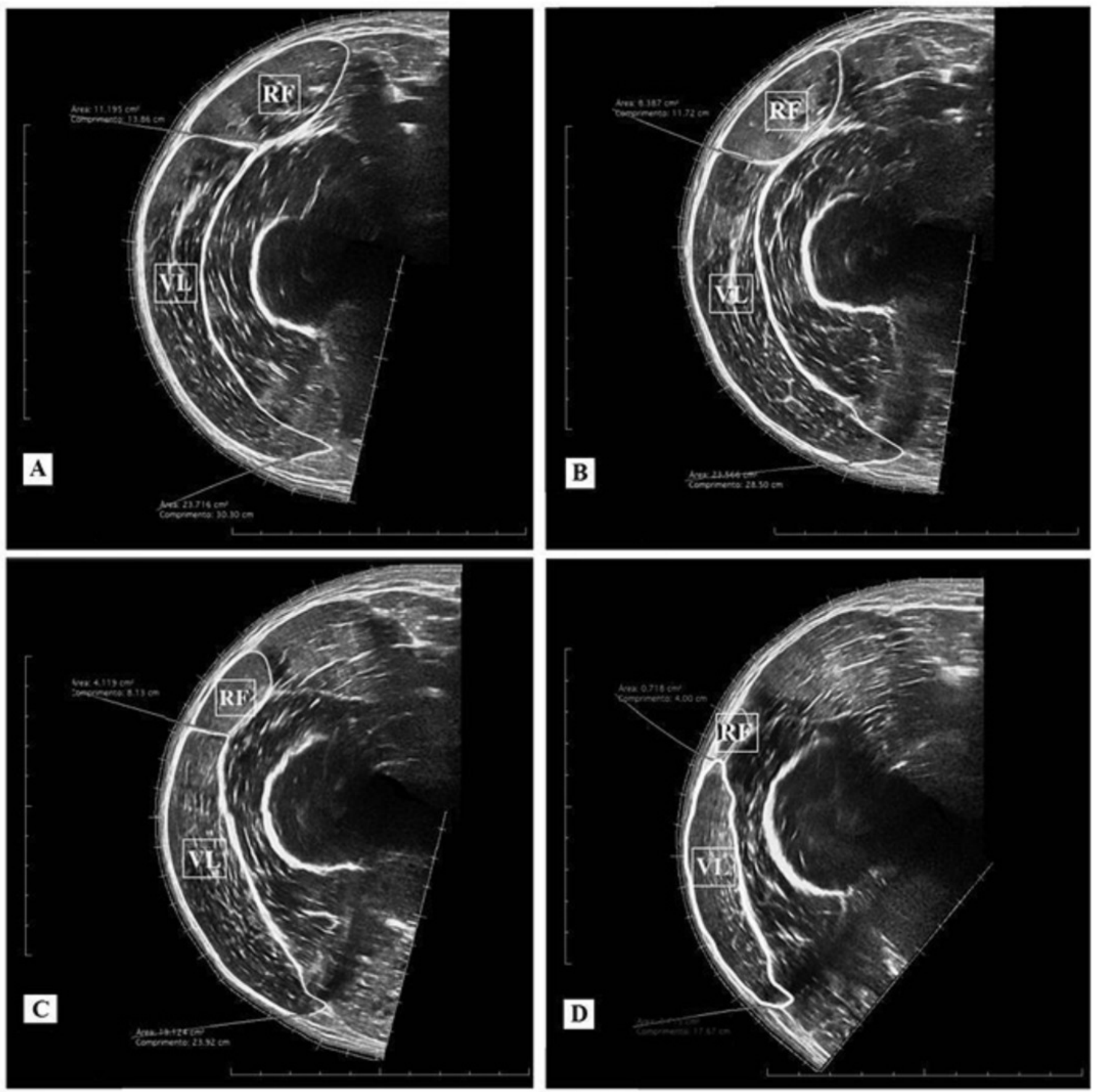
Figure 3

Rectus femoris (A) and vastus lateralis (B) muscle cross-sectional areas (CSA) at pretest to post-test for each training protocol.

Mean (vertical bars); standard deviation (vertical lines); individual values for each training protocol (black circles). " Post-test higher than pre-test for both protocols (time main effect). 


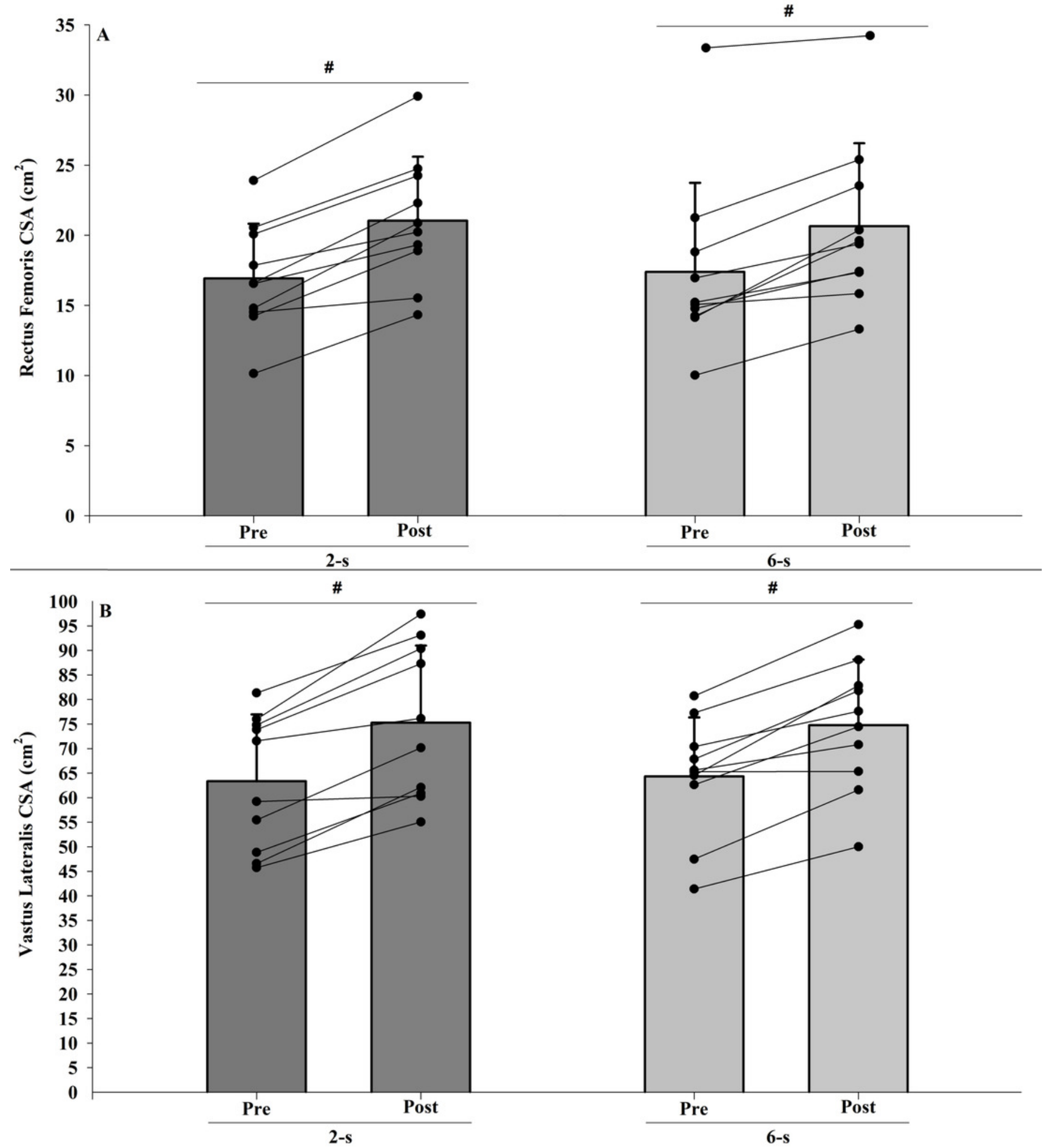


Figure 4

Maximal dynamic strength (1RM) test at post-test for each training protocol.

Mean (vertical bars); standard deviation (vertical lines); individual values for each training protocol (black circles). 


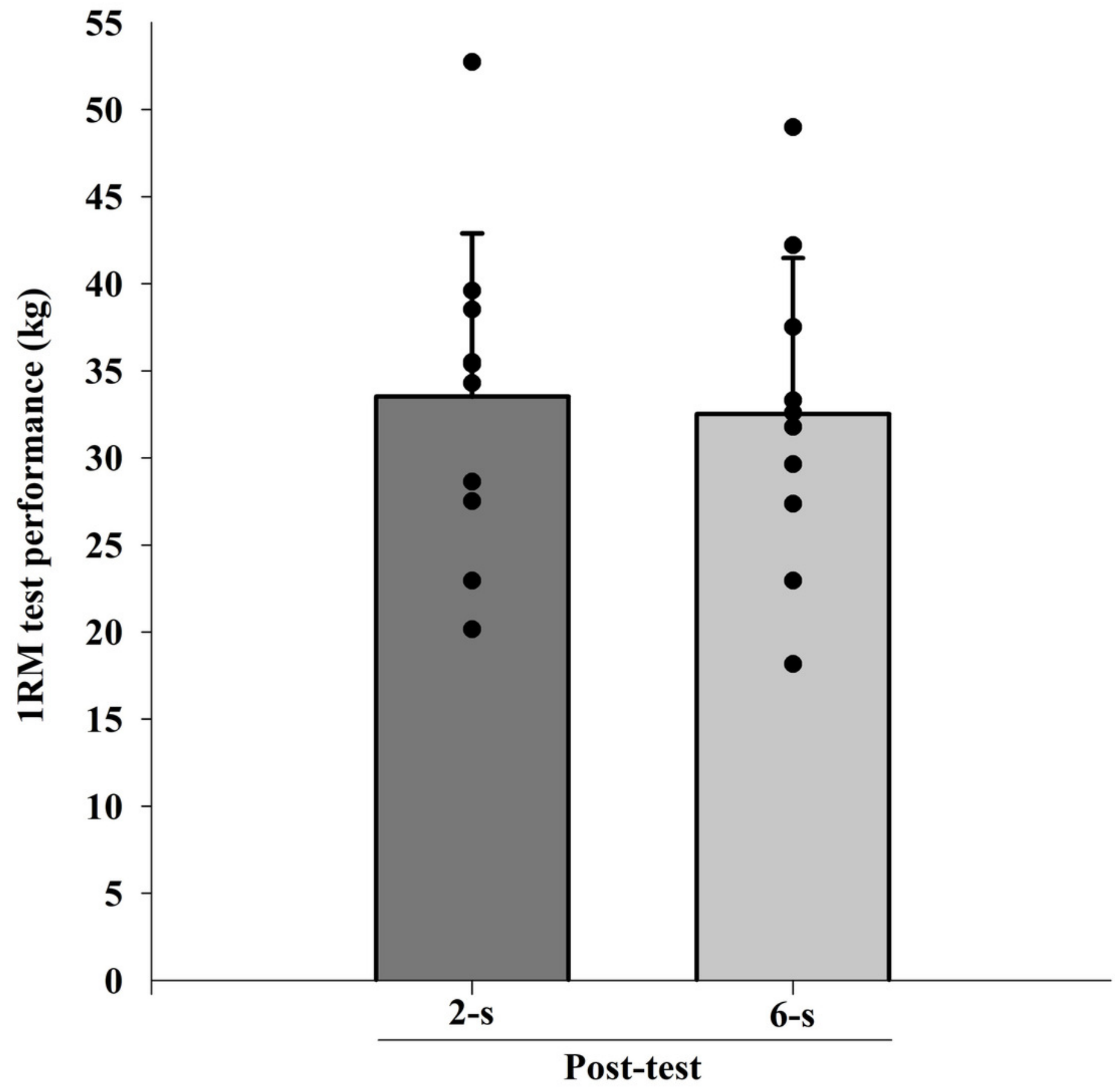




\section{Figure 5}

Maximal isometric strength (MVIC) at $30^{\circ}$ of knee-joint angle at pre-test to post-test for each training protocol.

Mean (vertical bars); standard deviation (vertical lines); individual values for each training protocol (black circles). * 6-s RD protocol higher than 2-s RD protocol. * Post-test higher than pre-test for both protocols (protocol and time interaction effect).
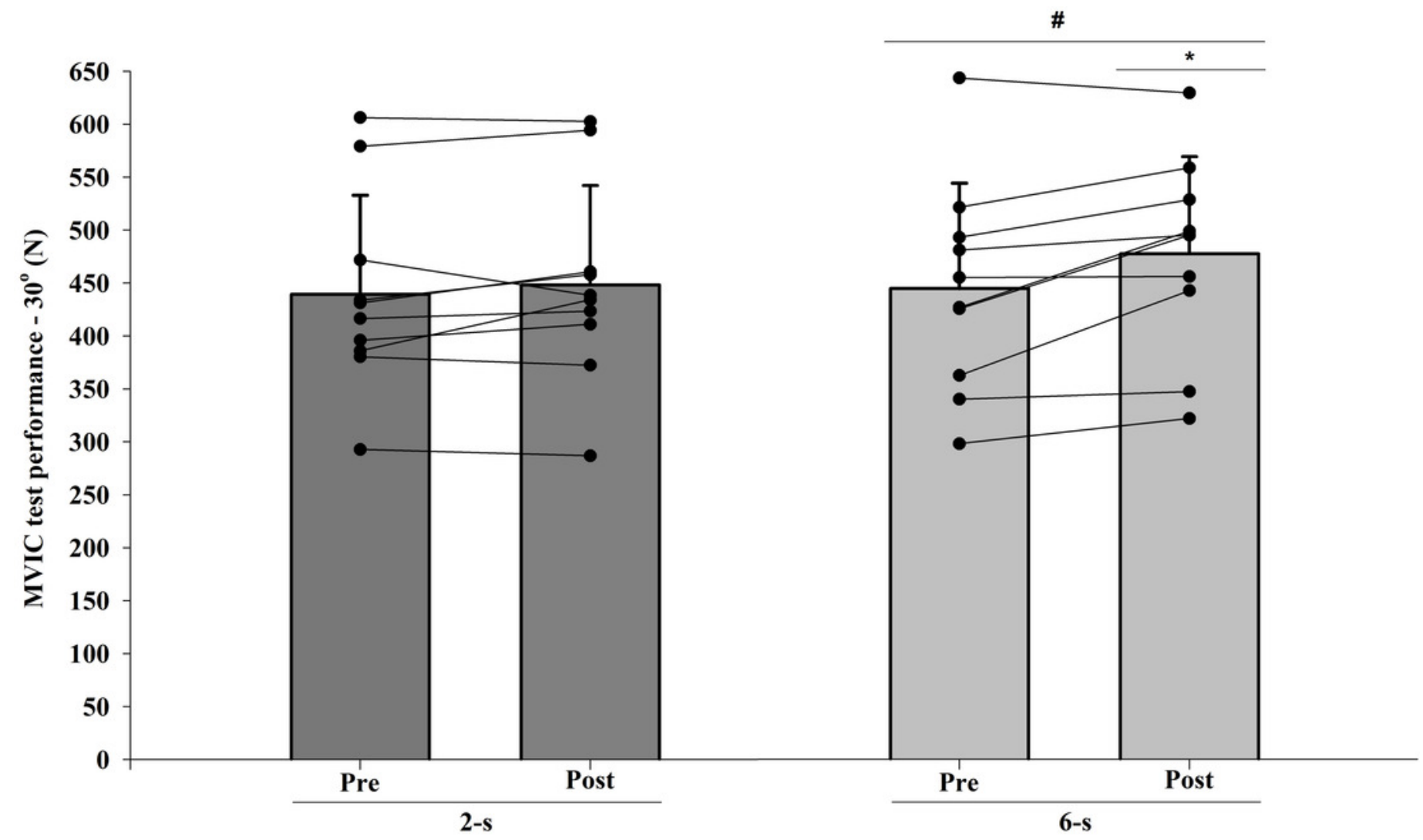
Figure 6

Maximal isometric strength (MVIC) at $90^{\circ}$ (B) of knee-joint angle at post-test for each training protocol.

Mean (vertical bars); standard errors (vertical lines); individual values for each training protocol (black circles). 


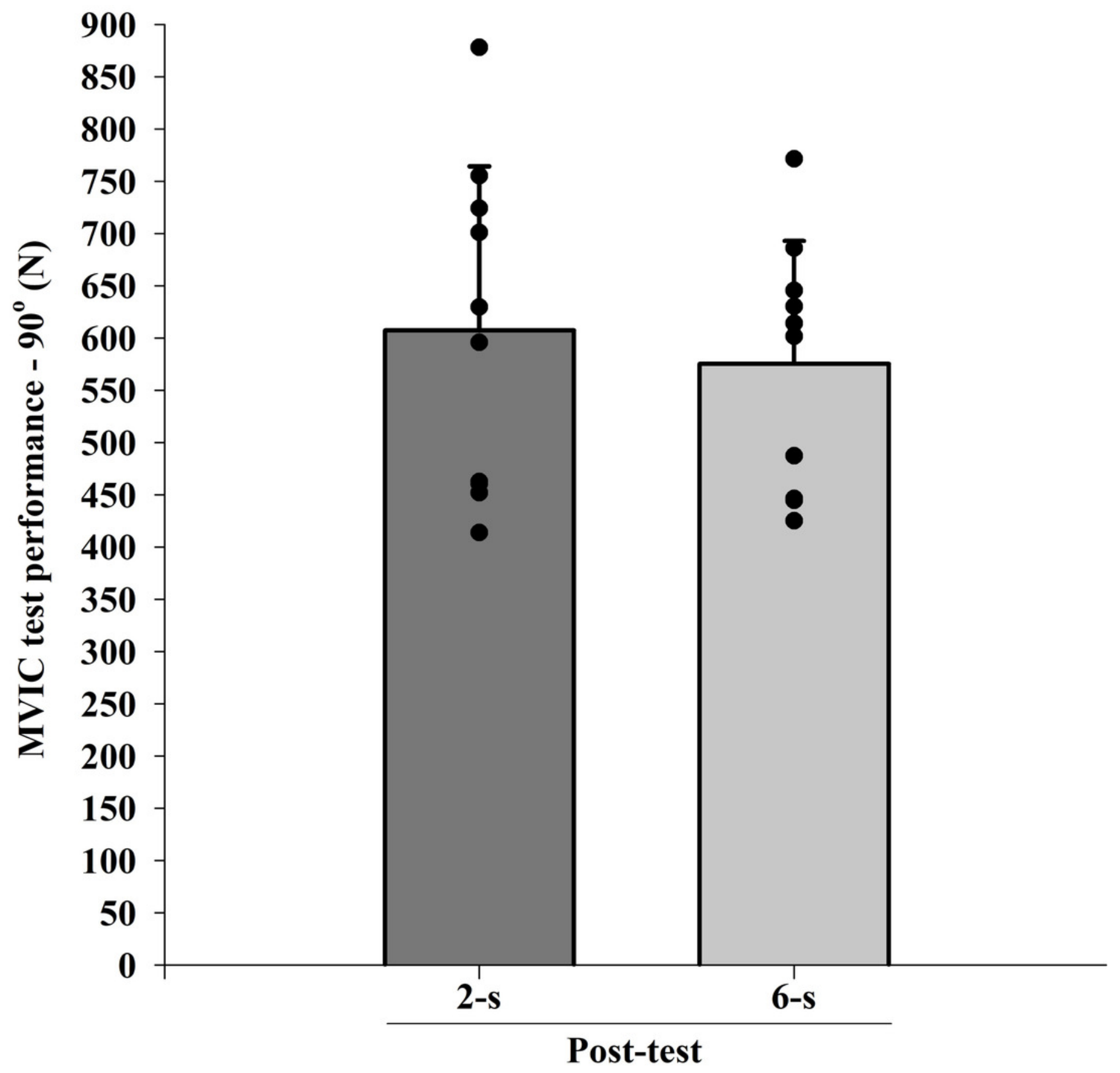


Figure 7

Rectus femoris concentric normalized EMG amplitude $\mathrm{x}$ knee-joint angle curves during $6^{\text {th }}(\mathrm{A})$ and $39^{\text {th }}(\mathrm{B})$ experimental sessions at 2-s and 6-s RD protocols.

Mean values for 2-s RD (white triangles); Mean values for 6-s RD (black circles); standard errors (vertical lines). * Significant difference between protocols. ${ }^{*}$ Higher than previous jointangle (6-s RD protocol). " Lower than all previous joint angles, except for $100-90^{\circ}$ (2-s RD protocol).
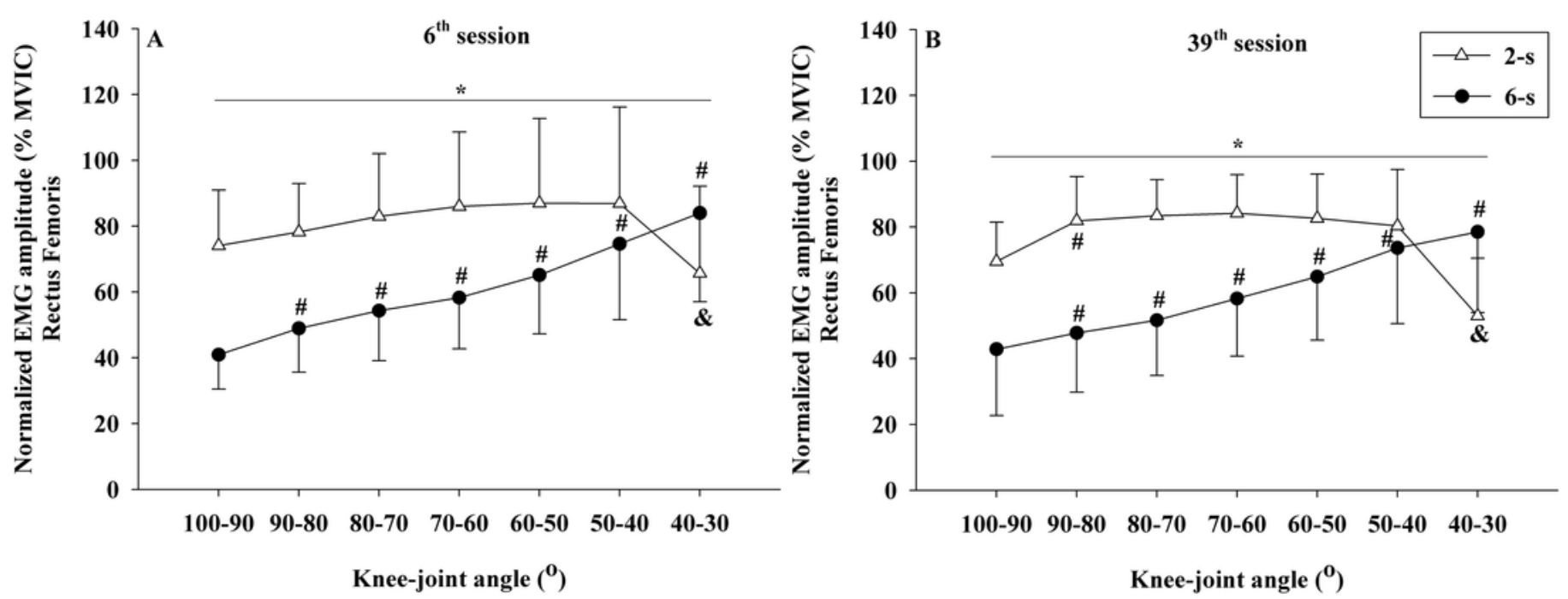
Figure 8

Vastus lateralis concentric normalized EMG amplitude $\mathrm{x}$ knee-joint angle curves during $6^{\text {th }}(A)$ and $39^{\text {th }}(B)$ experimental sessions at 2-s and 6-s RD protocols.

Mean values for 2-s RD (white triangles); Mean values for 6-s RD (black circles); standard errors (vertical lines). * Significant difference between protocols. " Higher than previous joint angle (6-s RD protocol). " Lower than all previous joint angles (2-s RD protocol).
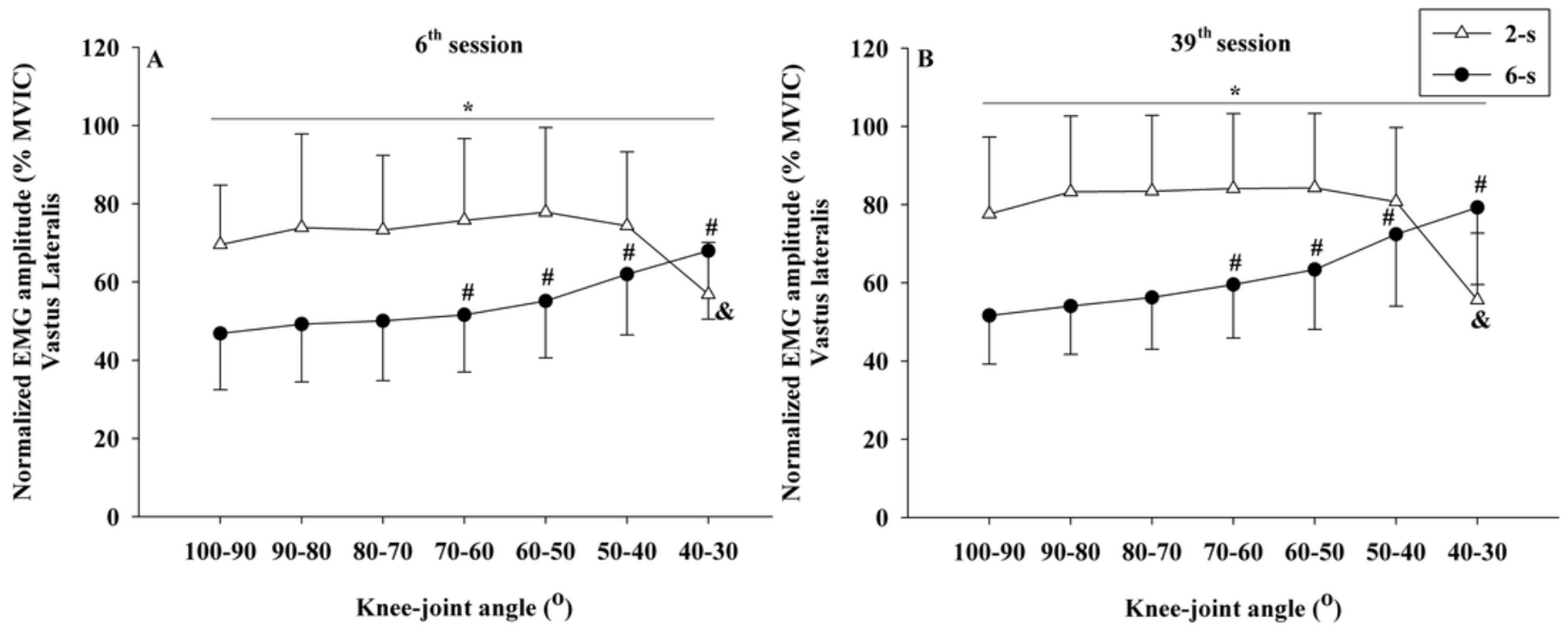
Figure 9

Concentric normalized force $x$ knee-joint angle curves during $6^{\text {th }}(A)$ and $39^{\text {th }}(B)$ experimental sessions at 2-s and 6-s RD protocols.

Mean values for 2-s RD (white triangles); Mean values for 6-s RD (black circles); standard errors (vertical lines). * Significant difference between protocols. ${ }^{*}$ Higher than previous joint angle (6-s RD protocol). " Lower than previous joint angle (2-s RD protocol).
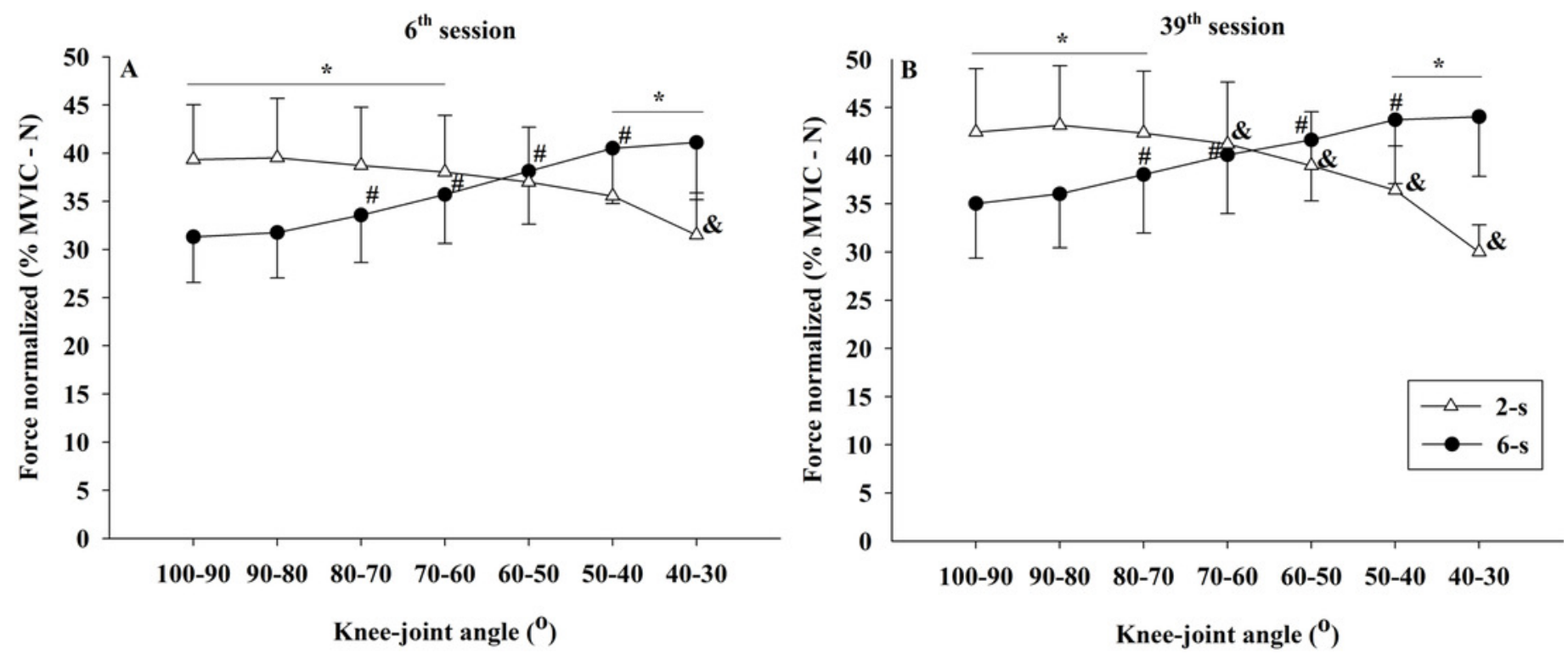